\title{
Enabling inference for context-dependent models of mutation by bounding the propagation of dependency
}

\author{
Frederick A Matsen IV ${ }^{2,3,4,5}$ and Peter L Ralph ${ }^{1, *}$ \\ ${ }^{1}$ Institute of Ecology 8 Evolution, Department of Biology, University of Oregon \\ ${ }^{2}$ Computational Biology Program, Fred Hutchinson Cancer Research Center, United States \\ ${ }^{3}$ Department of Genome Sciences, University of Washington \\ ${ }^{4}$ Department of Statistics, University of Washington \\ ${ }^{5}$ Howard Hughes Medical Institute \\ *Corresponding author: plr@uoregon.edu
}

December 15, 2021

\begin{abstract}
Although the rates at which positions in the genome mutate are known to depend not only on the nucleotide to be mutated, but also on neighboring nucleotides, it remains challenging to do phylogenetic inference using models of context-dependent mutation. In these models, the effects of one mutation may in principle propagate to faraway locations, making it difficult to compute exact likelihoods. This paper shows how to use bounds on the propagation of dependency to compute likelihoods of mutation of a given segment of genome by marginalizing over sufficiently long flanking sequence. This can be used for maximum likelihood or Bayesian inference. Protocols examining residuals and iterative model refinement are also discussed. Tools for efficiently working with these models are provided in an $\mathrm{R}$ package, that could be used in other applications. The method is used to examine context dependence of mutations since the common ancestor of humans and chimpanzee.
\end{abstract}

\section{Introduction}

Early models of DNA sequence mutation assumed that each nucleotide was equally likely to mutate into any other nucleotide [Jukes and Cantor, 1969]. These were followed by others that incorporated differences in the mutation process, such as higher transition to transversion ratio [Hasegawa et al., 1985, Felsenstein and Churchill, 1996]. Although the rates of mutation of a nucleotide are known to depend on not only the nucleotide itself but also nearby sequence context [Seplyarskiy et al., 2021], it is difficult to take this context into account when performing inference.

This situation from biological sequence analysis is an example of a more commonly encountered class of models in statistics: a lattice of sites, with each site taking one of a finite collection of possible states, and whose stochastic, temporal evolution is Markov and governed by a set of local rules. For instance, at each site may sit a nucleotide whose mutation rate depends on physical properties determined by the nearby DNA sequence; a cell whose infection status depends on the state of its neighbors; or a particle whose spin is perturbed by external noise to a state whose distribution depends on the local energy configuration. Practical use of such models often requires inferring transition rules based on observations of the system at several time points, or of several states evolved along a tree from a single starting point. This paper concerns scalable methods for doing inference under such models using observations.

The situation is similar to the widely-studied problem of inference based on observation of a single instance of a Markov random field, and in many cases reduces to this if we only observe the system at one time point at stationarity. These are used, for instance, in spatial statistics [Besag, 1972, Gelfand et al., 2010] and 
image reconstruction [Geman and Geman, 1984, Besag, 1986]. The conditioning method we consider here is similar to the "coding" scheme introduced by Besag [1974], that conditions on a set of sites that makes the remaining observations independent; consistency of such methods has been shown by Comets [1992] and reviewed by Larribe and Fearnhead [2011]. A good review of recent statistical techniques is given by Friel [2012].

In the context of genomics, it is well-known that certain short nucleotide sequences are in many organisms much more, or less, abundant than expected by chance [Burge et al., 1992], due to the combined effects of context dependence of the nucleotide mutation process, selective constraints on the function of the sequence, and other processes such as biased gene conversion [Duret and Galtier, 2009, Arbeithuber et al., 2015]. Indeed, molecular studies have demonstrated that the spectrum of new mutations in humans is highly context dependent [Schaibley et al., 2013, Gao et al., 2019, Rodriguez-Galindo et al., 2020] and that multinucleotide substitutions are relatively common [Schrider et al., 2011, Terekhanova et al., 2013, Harris and Nielsen, 2013]. In humans, the molecular processes underlying mutations are best studied in contexts of intense somatic mutation: cancer and the hypervariable regions that generate immune system diversity [Cobey et al., 2015, Heredia-Genestar et al., 2020], which provide quite different cellular contexts but can share commonalities due to shared molecular machinery. In general, substitution rates depend on sequence context due to the interaction of a great many factors including: (a) likelihood of DNA damage or errors in cell division (e.g., UV damage [Goodman, 2002] or crossing over in meiosis [Arbeithuber et al., 2015]); (b) activity levels and structural properties of the enzymes responsible for repair (e.g., Y-family polymerases [Goodman and Woodgate, 2013, Sale et al., 2012]); and (c) any functional effects of an error (e.g., lethal mutations will not be seen in a population census). We are concerned with effects that are homogeneous across the sequence, so do not consider further the many constraints on protein-coding sequence [reviewed in Thorne, 2007]. Some of these effects - particularly, activity levels of different enzymes in the germline - will likely vary over evolutionary time, and so comparisons between widely separated species will identify time-averaged mutation rates. Studies have used a variety of exploratory techniques to identify sets of mutational patterns co-occurring in comparisons (a) between different cancer types [Alexandrov et al., 2013b, Shiraishi et al., 2015] and (b) between different human populations [Harris, 2015, Harris and Pritchard, 2016, Mathieson and Reich, 2017], to disentangle distinct signals presumably coming from the action of distinct sources of mutation.

However, phylogenetic and population genetic methods usually ignore such dependencies in the interest of computational efficiency, but some progress has been made. Often, the effects of a complex mutational spectrum are explored using summary statistics of larger blocks or other sensible but ad hoc methods. For instance, Arndt et al. [2003] studied dinucleotide transitions, and Yaari et al. [2013] displayed strong heterogeneity in the probability of synonymous mutations across 5-mers in B-cell immunoglobulin genes. Others have made progress using simplifying assumptions [Bérard and Guéguen, 2012], or by other approximations [Christensen et al., 2005, Saunders and Green, 2007]. Pedersen and Jensen [2000], later extended by Hwang and Green [2004], Hobolth [2008] and Baele et al. [2010a], used data augmentation and an MCMC algorithm to do Bayesian inference; Lunter and Hein [2004] used an approximate matrix decomposition; while Siepel and Haussler [2004] and later [Baele et al., 2010b] computed likelihoods by assuming a model that is Markov along the genome. However, all these methods are quite computationally intensive.

Another canonical example of context-dependent transition is the Ising model of statistical physics with time evolution given by Glauber dynamics [Glauber, 1963], in which a lattice of up/down spins are perturbed by thermal noise, relaxing into states dependent on the energy of the resulting configuration. Parameter estimation for Ising model without temporal dynamics is relatively well-understood [Pickard, 1982, Frigessi and Piccioni, 1990], but the problem of dynamical observations is less well-studied.

The general framework also fits certain cellular automata models, e.g., modeling wildfire [Clarke et al., 1994], the spread of HIV [Zorzenon dos Santos and Coutinho, 2001], or land use patterns [Wu, 2002]. Complex models may introduce long-range dependencies beyond the scope of this paper, but these methods may still prove useful in the modeling process.

This general class of models are known in the probability literature as interacting particle systems [Liggett, 2005] with neighborhood structure - continuous-time Markov chains on lattice-indexed collections of states 


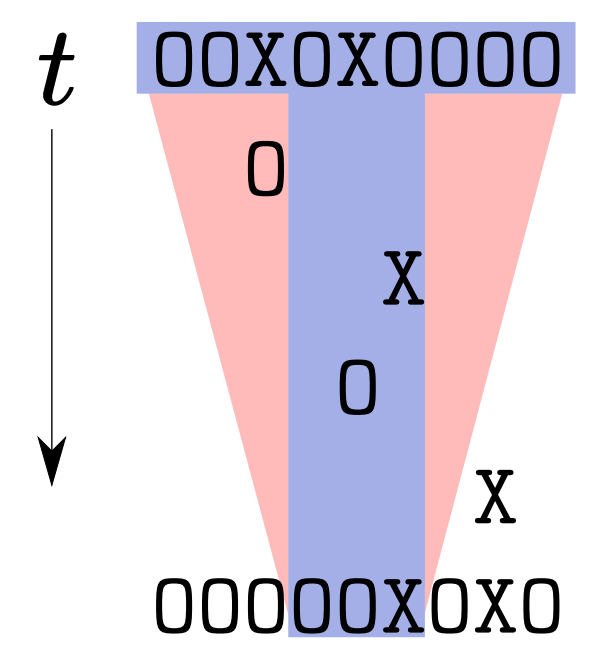

Figure 1: An overview of the method for a neighbor-dependent model, showing an initial sequence on top, a collection of mutations progressing through time, and a final sequence. The pink trapezoidal region represents the "range of influence", i.e., possible locations and times at which a mutations is likely to affect the mutation outcome at the sites of interest (the three sites in the center of the final sequence). The blue $\mathrm{T}$ shape is called a T-mer, which is how our approximation to the process is parameterized. The central, smaller segment of the $\mathrm{T}$ is called the base of the T-mer, and the key building block computes the transition probability for this subsequence conditioned on the ancestral state at the additional overhang sites of the top of the $\mathrm{T}$.

whose transition probabilities are local in the sense that any instantaneous change only affects a small number of nearby sites, and the rates of such instantaneous changes depend only on the states in some bounded neighborhood of those sites to change. As finite-state Markov chains, transition probabilities are in principle simply expressable as a matrix exponential, but this is impractical because the size of the matrix is equal to the number of possible configurations.

In this paper, we propose a solution to this problem, showing that the conditional likelihood of local pattern count statistics can be well-approximated by marginalizing over a finite amount of surrounding context. We then use this to conjecture an approximation for the full likelihood. Motivated by the problem of inferring context-dependent mutation rates from diverged nucleotide sequences, we extend the inference framework to observations on trees. The tools are available as an $\mathrm{R}$ package, which is fairly efficient thanks to computational techniques using sparse matrices.

Before diving into a formal problem specification, we offer an intuitive explanation of how the method works. The difficulty of context-sensitive models is that, in principle, one may have a sequence of events with cascading consequences that involves a lot of the sequence. In the extreme case, one may have a sequence of mutations from, say, the left end of the sequence to the right end of the sequence, such that the probability of each mutation has been changed by that of the previous one.

However, the starting point of the work presented here is that for typical models such a sequence of events is improbable. For that reason, we can compute the transition probability for each segment of the sequence using a local context which, although bigger than the context which determines the local transition rates, is much smaller than the entire sequence. We call the building blocks of this computation T-mers (Figure 1) which parameterize transition probabilities for a small section of sequence (the base of the T-mer) based on additional sequence (the overhang of the T-mer). We can then bound the probability that the influence of a mutation outside the overhang of the T-mer will impact the sites at the base of the T-mer, which translates into an estimate of approximation error in our calculation of the likelihood. 


\section{A general context-dependent model}

Consider a 1-dimensional grid of sites, each of which can take one of a finite set of states, and that switch randomly between states according to a local set of rules. We then observe a finite collection of these sites at only a few times, Suppose that the dynamics are Markov: writing $\mathcal{S}$ for the set of possible states and $X_{i}(t)$ for the state of site $i$ at time $t$, we assume that $\{X(t)\}_{t \geq 0}$ is a Markov process on sequences of $L$ states $\mathcal{S}^{L}$ for which the probability a given site changes state in a small amount of time depends only on the sequence of states at nearby sites.

To formalize this notion of local dependence, first define a pattern to mean a contiguous sequence of states, and let $|u|$ denote the length of a pattern $u$. Given a sequence $x \in \mathcal{S}^{L}$, let $x_{i}^{(h)}=\left(x_{i}, x_{i+1}, \ldots, x_{i+h-1}\right)$ denote the pattern of $x$ of length $h$ beginning at location $i$. The dynamics of our stochastic process are determined by the set of allowed transitions and associated rates: given two patterns $u$ and $v$ of common length $h$, saying "pattern $u$ changes to $v$ at rate $\mu$ " means that

$$
\mathbb{P}\left\{X_{i}^{(h)}(t+d t)=v \mid X_{i}^{(h)}(t)=u\right\}=\mu d t+o(d t),
$$

regardless of the location $i$ along the sequence. If more than one pattern would match to cause the same change, their rates add. Such a rule is defined by its "transition triple" $(\mu, u, v)$, written as " $u \rightarrow v$ at rate $\mu$ ". A model of context-dependent mutation is defined by a set of all transition triples, which we denote $\mathcal{T}$.

Although our main focus is on nucleotides, it is informative to consider other, simpler, models as well. Here is one such example.

Example 1.1 (TASEP). In the Totally Asymmetric Simple Exclusion Process describes a set of particles moving among some sites, with at most one particle per site. Each site is either "empty" or "occupied" (0 or 1 , respectively), and each particle, independently at rate $\lambda$, checks whether the site to the right is empty, and if so, moves there. This process therefore has only one transition triple:

$$
10 \rightarrow 01 \text { at rate } \lambda
$$

This only has one parameter. If we allow particles to exit from the right and new particles to enter from the left (e.g., by adding special symbols for the start and end of the sequence), then from observing only starting and ending configurations we cannot tell with certainty which particles have moved where, so it is not obvious how to estimate the speed. However, summing over possible particle movements would allow us to compute the likelihood of a given configuration change, and so estimate the speed by maximum likelihood given an initial sequence, a final sequence and an elapsed time.

To allow more compact specification of models, we also introduce a "potential": suppose that there is a collection of patterns $\left\{p_{i}\right\}$ such that each occurrence of $p_{i}$ adds a quantity $e_{i}$ to the total "energy" of a sequence, and that the rate at which each possible change occurs is modulated by a function of the energy difference the change would produce. This is natural if transition triples describe proposed changes, and that the probability a proposed change actually occurs depends on how much it affects the energy.

Concretely, let $\mathcal{P}=\left\{\left(p_{i}, e_{i}\right)\right\}$ be a set of (pattern, energy difference) pairs, and for any sequence $x$ define $E(x)=\sum_{i} e_{i} n\left(x, p_{i}\right)$, where $n\left(x, p_{i}\right)$ is the number of times the pattern $p_{i}$ occurs in $x$. These will affect the rates through a nonnegative function $\phi$ : we declare that if the rate at which $x$ changes to $y$ as computed from transition triples is $\mu$, then the actual rate is

$$
\mu \phi(E(y)-E(x)) .
$$

If $\phi(e)$ is not greater than 1 , one way to think about this is that the transition triples give "proposed changes", but these changes only take effect with probability $\phi(E(y)-E(x))$. We have described this idea in terms natural for a physics model in which high energy configurations are disfavored, however it is also suitable for a population genetics model in which $\phi$ describes the probability of fixation of a mutant allele given a certain fitness change from the parent. 
Example 1.2 (Genomic GC content). A genome sequence can be written using A, C, G, and T; in the most general model of independent mutation across sites, each of the 12 possible transitions occurs at its own rate. Furthermore, in many species adjacent, methylated CG dinucleotides ("CpG sites") have a much higher mutation rate to TG and CA than either single nucleotide change under the independent model. Embellishing the single-nucleotide model with this additional rate results in the model defined by

$$
\begin{array}{ccccc}
x & \rightarrow y & \text { at rate } & m_{x y} & \text { for } x, y \in\{\mathrm{A}, \mathrm{C}, \mathrm{G}, \mathrm{T}\} \text { and } x \neq y \\
\mathrm{CG} & \rightarrow \mathrm{TG} \text { at rate } \gamma \\
\mathrm{CG} & \rightarrow \mathrm{CA} \text { at rate } \gamma
\end{array}
$$

where $\gamma$ is the additional $\mathrm{CpG}$ rate above the base mutation rate. Note that for a given pair of sequences, a change ACG $\rightarrow$ ATG could have occurred by a $\mathrm{C} \rightarrow$ T mutation via the independent-sites model, or by a $\mathrm{CG} \rightarrow$ TG mutation; in such cases the total instantaneous rate at which ACG changes to ATG is the sum of the rates (in this example, $\gamma+m_{\mathrm{CT}}$ ).

Counteracting this trend towards increased G/C is GC-biased gene conversion [Glémin et al., 2015], which acts effectively as a selective pressure against $\mathrm{G}$ or $\mathrm{C}$ bases. We model the evolution of a single sequence, not a population of sequences, imagining this sequence to be the consensus sequence of the population. A new mutation with an effect $s$ on fitness that occurs in a population of effective size $N_{e}$ becomes ubiquitous, rather than dying out, with probability approximately $(1-\exp (-2 s)) /\left(1-\exp \left(-2 s N_{e}\right)\right)$ [Kimura, 1962]. If the mutation occurs at rate $\mu$ per individual, the total rate it occurs at in the population is $\mu N_{e}$. GC-biased gene conversion effectively means that sequences with more G/Cs are selected against. This is incorporated as follows: set the energy of patterns $\mathrm{C}$ and $\mathrm{G}$ to be $s$, so that $E(y)-E(x)$ is the net change in number of G's and C's multiplied by $s$. Also, define $\phi(e)$ to be the expected rate of fixations of "selected" mutations with advantage $e$ relative to the per-individual, per-site mutation rate:

$$
\phi(e)=N_{e}(1-\exp (-2 e)) /(1-\exp (-2 e N)) .
$$

With these definitions, if a given change that occurs with rate $\mu$ would change a sequence $x$ to $y$, then $\mu \phi(E(y)-E(x))$ is the rate at which the mutation appears and successfully takes over in the population.

We close with a final example from statistical physics, to be used later:

Example 1.3 (Gibbs sampling of the Ising model). In the Ising model, each site is labeled as either "up" or "down" ( +1 or -1 respectively), imagined as a string of $n$ magnetic dipoles, and the energy associated with a given state $x$ is $E(x)=-\frac{1}{2} \beta \sum_{i=1}^{n-1} x_{i} x_{i+1}-\frac{1}{2} \gamma \sum_{i=1}^{n} x_{i}$. Here $\beta$ represents inverse temperature, and $\gamma$ represents the strength of the magnetic field (here scaled by temperature). The associated stationary distribution on configurations is proportional to $\exp (-E(x))$. The following process, also known as "Glauber dynamics", preserves the stationary distribution: each site, independently at rate $\lambda$, forgets its spin, and reconfigures to a state chosen with probability proportional to the stationary probability of the resulting configuration: Since $\exp (-E(x)) /(\exp (-E(x))+\exp (-E(y)))=1 /(1+\exp (-(E(x)-E(y)))$, this is:

$$
\begin{aligned}
& +\rightarrow-\text { at rate } \lambda \quad \text { pattern energy } \\
& -\rightarrow+\text { at rate } \lambda \text { and } \quad+- \text { or }-+\quad \beta
\end{aligned}
$$

and

$$
\phi(e)=\frac{1}{1+\exp (-e)}
$$

The generator matrix We now describe concretely how the set of transition rates on patterns determines the transition rate matrix for complete sequences, that is, the $|\mathcal{S}|^{n} \times|\mathcal{S}|^{n}$ matrix $G(n)$ whose $(x, y)^{\text {th }}$ entry gives the instantaneous rate with which the process in state $x \in \mathcal{S}^{n}$ jumps to state $y \in \mathcal{S}^{n}$. Recall that 
$x_{i}^{(h)}=\left(x_{i}, x_{i+1}, \ldots, x_{i+h-1}\right)$ denotes the subsequence of length $h$ beginning at location $i$. For each $1 \leq i \leq n$, pattern length $h>0$, and patterns $u, v \in \mathcal{S}^{h}$ define the relation

$$
x \stackrel{i, u, v}{\longrightarrow} y \quad \text { iff } \quad \begin{cases}x_{k}=y_{k} & \text { for } k<i \\ x_{i+k}=u_{k} & \text { for } 0 \leq k<h \\ y_{i+k}=v_{k} & \text { for } 0 \leq k<h, \text { and } \\ x_{k}=y_{k} & \text { for } k \geq i+h,\end{cases}
$$

i.e., if $x$ and $y$ match except at positions $i, i+1, \ldots, i+h-1$, and for those positions $x$ matches with $u$ while $y$ matches with $v$.

As before, let $\mathcal{T}$ be the set of transition triples for the model. As noted above, there may be more than one way to mutate a sequence $x$ to get $y$ : for each position $i$, let $J(i, x, y)$ be those transitions in $\mathcal{T}$ that can be applied at $i$ to change $x$ into $y$, i.e.,

$$
J(i, x, y)=\{(\mu, u, v) \in \mathcal{T}: x \stackrel{i, u, v}{\longrightarrow} y\} .
$$

The rate $G(n)_{x, y}$ is then the sum of all transition rates that can take $x$ to $y$, multiplied by the fitness term, namely

$$
G(n)_{x, y}=\phi(E(y)-E(x)) \sum_{i=1}^{n} \sum_{(\mu, u, v) \in J(i, x, y)} \mu
$$

and if there are no triples $(\mu, u, v)$ with $x \stackrel{i, u, v}{\longrightarrow} y$ for some $i$, then $G(n)_{x, y}=0$. To make this the generator matrix for a Markov process, we also set each $G(n)_{x, x}$ so that rows sum to zero. Indeed, this matrix is very sparse because for most pairs $(x, y)$ there are no transition triples that can change $x$ into $y$.

In principle, this gives us the transition probabilities for the process by a matrix exponential:

$$
p_{n}(t ; x, y):=\mathbb{P}\{X(t)=y \mid X(0)=x\}=\left(e^{t G(n)}\right)_{x y},
$$

which would then provide a route to parameter estimation. In practice, the size of these matrices makes direct application obviously impractical for anything but very small $n$. This paper proposes an alternate strategy.

\section{Simulation}

First, it will be useful for later proofs to have an explicit construction of the process, also used for simulation, using a variant of the "jump chain representation", also known as the "Gillespie algorithm" or "uniformization" [Hobolth and Stone, 2009]. Briefly, this works by first sampling a homogeneous Poisson process of times and locations of possible changes, at an appropriate rate $\mu^{*}$ per site, and then resolving each possible change in temporal order. (Note that these "possible changes" are different than the "proposed changes" described above in terms of the energy model.) The rate $\mu^{*}$ should be the maximum "local" rate at which transitions occur, across sites and transition outcome states:

$$
\mu^{*}=\max _{x} \sum_{y \neq x} G(L)_{x, y} .
$$

Note that we do not actually need to construct $G(L)$ to find the maximum rate. Now sample possible changes: in a sequence of length $L$, over a time period $t$, first draw the number of possible changes, $N$, from a Poisson distribution with mean $\mu^{*} t L$, and then choose the times $s_{k}$ and locations $i_{k}$ at which possible changes occur uniformly over $[0, t]$ and $\{1,2, \ldots, L\}$ respectively. Order the possible change times, so that

$$
0<s_{1}<\cdots<s_{N}<t .
$$


(Note that this is equivalent to saying that possible changes occur at rate $\mu^{*}$ independently at each of the $L$ sites.) Now, suppose we have determined the state just before time $s_{k}$ to be $X\left(s_{k-1}\right)=x$. Then, the new state $X\left(s_{k}\right)$ is chosen by applying a mutation at position $i_{k}$ with probability proportional to the corresponding rate, i.e., out of the possible transitions $\mathcal{T}$, transition triple $(\mu(j), u(j), v(j))$ is chosen with probability

$$
q_{j}= \begin{cases}\mu(j) / \mu^{*} & \text { if } x_{i}^{(|u(j)|)}=u(j) \\ 0 & \text { otherwise. }\end{cases}
$$

The remaining probability $q_{0}=1-\sum_{j=1}^{|\mathcal{T}|} q_{j}$ gives the probability that the state remains the same. (By construction of $\mu_{*}, q_{0} \geq 0$.) If triple $(\mu(j), u(j), v(j))$ is chosen, then the substring of $x$ matching $u(j)$ that begins at position $i_{k}$ is replaced with $v(j)$ to produce $X\left(s_{k}\right)$.

For instance, to simulate the TASEP, one has only to let $\mu^{*}=\lambda$, and at each possible change check if at site $i_{k}$ there is a 1 followed by a 0 , and if so, switch them.

\section{Inference}

The problem at hand is to infer the parameters of the model based on the final state of the model after it has evolved from some known initial state. How can we extract the information? These are Markov processes, on the state space $\mathcal{S}^{L}$, so the full likelihood function is given by (2), but doing anything with the $|\mathcal{S}|^{L} \times|\mathcal{S}|^{L}$ matrix $G_{L}$ is clearly infeasible even for moderately sized $L$. We can, however, compute (2) with smaller $G_{n}$, so the first thing that one might think to do is to break the sequence up into many blocks of length $m$, and treat these as independent. Then, defining $p_{m}(t ; x, y)$ to be the probability that a string $x$ of length $m$ evolves to string $y$ over time $t$, we would obtain the approximate likelihood function

$$
\prod_{k=0}^{n / m} p_{m}\left(x_{k m+1}^{(m)}, y_{k m+1}^{(m)}\right) .
$$

However, this ignores dependencies between neighboring blocks, so it is not clear how good this approximation might be.

The key insight we use is that by including neighboring sequence context in the initial sequence only, we can compute something correctly, and that will be sufficient to compute the full likelihood, at least arbitrarily. To do this, let $p_{n, \ell, r}(x, y)$ for $\ell+r<n$ denote the probability that an initial subsequence $x$ of length $n$, after time $t$, is found to match a smaller subsequence $y$ of length $n-\ell-r$, offset by $\ell$ sites (see Figure 2). This central, smaller subsequence is called the "base" of the T-mer. We refer to such patterns as T-mers, as depicted in Figure 2. We emphasize that the overhang of the T-mers is typically bigger than the context-sensitivity width of the underlying model (Figure 1).

These $p_{n, \ell, r}(x, y)$ probabilities can be found by marginalizing $p_{n}$, summing over the possible initial and final substrings of $x$ (denoted $a$ and $b$ ):

$$
p_{n, \ell, r}(t ; x, y)=\sum_{a \in \mathcal{S}^{\ell}} \sum_{b \in \mathcal{S}^{r}} p_{n}(t ; x, a \oplus y \oplus b), \quad \text { for } x \in \mathcal{S}^{n}, y \in \mathcal{S}^{n-\ell-r},
$$

where $a \oplus y \oplus b$ is the sequence composed of concatenating $a, y$, and $b$ together in that order. We expect $p_{n, \ell, r}(x, y)$ to be asymptotically correct when $\ell$ and $r$ are large (and $t$ is fixed). In Appendix $\mathrm{B}$, we show that this is so, namely that the probability a given subsequence of length $n$ matching $x$ is later observed to have its center matching $y$ is close to $p_{n, \ell, r}(x, y)$, regardless of wider context. Formally, for each $i$,

$$
\mathbb{P}\left\{X_{i+\ell}^{(n-\ell-r)}(t)=y \mid X_{i}^{(n)}(0)=x\right\} \approx p_{n, \ell, r}(x, y),
$$

with the approximation getting better for longer $\ell$ and $r$. 
(A)

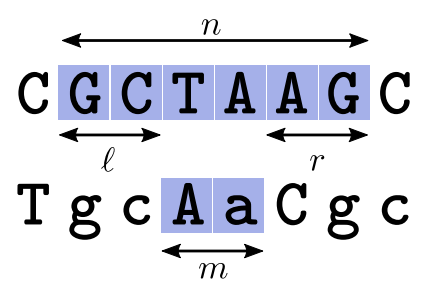

$\mathrm{A}(n, \ell, r) \mathrm{T}$-mer.
(B)

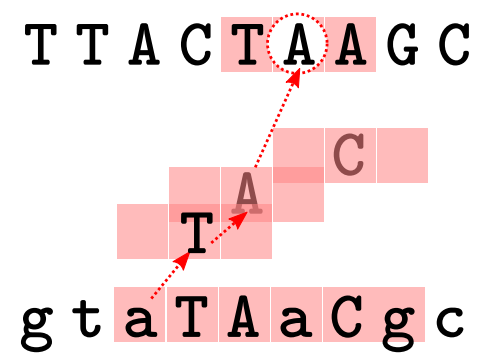

(C)

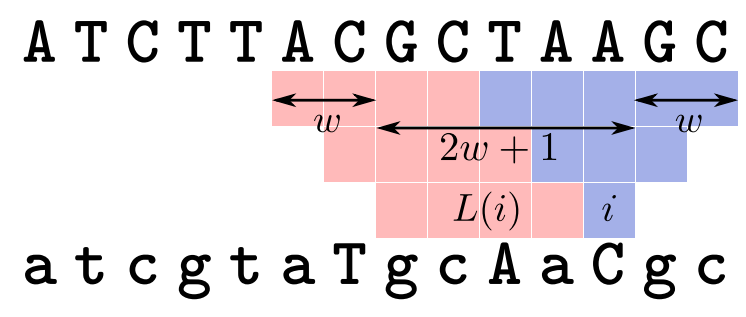

Figure 2: (A) A $(6,2,2)$ T-mer. Nonmutated sites are shown in lower case letters. The "base" of the T-mer is the lower central string of length $m=n-\ell-r$. (B) The three mutations have extended the influence of the circled $\mathrm{A}$ in the initial sequence (top) to the six positions colored red in the final sequence. The rate of each mutation depends on its' neighboring sites, i.e., with a window of width $w=1$. Red dotted arrows show the propagation of dependency between the circled A and the third site in the final sequence (bottom). (C) Diagram of heuristic argument for full likelihood approximation: site $i$ depends on the blue squares in $N_{i}$; the sites to the left of this in $L(i)$ are those other sites in $X(t)$ whose dependency neighborhoods overlap $N_{i}$. Red squares give the dependency neighborhood of $L(i)$, so we condition on the row of red and blue sites at the top. On the bottom, we have $2 w+1$ sites; at the top we have $4 w+1$; here $w=2$. 
Equation (3) could then be used to compute a composite likelihood, which we denote $A_{n, \ell, r}(x, y)$. This is the product of the probabilities of transition for all $(n, \ell, r)$ T-mers between $x$ to $y$, i.e., with $m=n-\ell-r$,

$$
A_{n, \ell, r}(x, y)=\prod_{i=1}^{L-m+1} p_{n, \ell, r}\left(x_{i}^{(n)}, y_{i+\ell}^{(m)}\right) .
$$

To keep notation simple we've ignored the boundaries of the sequence; to deal properly with these, on the right-hand side of the equation $\ell$ should be replaced with $\min (\ell, \max (i-1,0))$, and $r$ should be replaced with $\min (r, \max (n-i-\ell-m+1,0))$.

\subsection{Full likelihood}

What we actually want is the full likelihood $\mathcal{L}(y \mid x)$, the probability that a given sequence $x$ evolves into another, $y$, over a given period of time. This full likelihood can be computed as the product of the probability of each site in $y$ given $x$ and all sites in $y$ further to the left. Defining $x_{<i}=\left(x_{1}, \ldots, x_{i-1}\right)$ to be the subsequence to the left of $i$, this is:

$$
\mathcal{L}(y \mid x)=\prod_{i=1}^{L} \mathbb{P}\left\{Y_{i}=y_{i} \mid Y_{<i}=y_{<i} \text { and } X=x\right\} .
$$

Each term in this product is the probability of $Y_{i}$, given all sites in $X$ and all sites in $Y$ to the left of $i$. It seems reasonable that, similar to equation (4) above, the process at position $i$ should be approximately independent of distant positions, given the neighboring positions.

Here is a heuristic argument, depicted in Figure 2C. Suppose that $\ell$ is long enough that approximation (4) holds with $\ell=r$. Then we know that site $i$ approximately depends only on mutations within distance $\ell$. Furthermore, the sites up to distance $2 \ell$ to the left of $i$ also depend on these mutations. We can now use (4) to restrict the dependency on $x$. Recall that the last base of $x_{i}^{(h)}$ is $x_{i+h-1}$. So, $x_{i-3 \ell}^{(4 \ell+1)}$ runs from $i-3 \ell$ to $i+\ell$, inclusive, while $y_{i-2 \ell}^{(2 \ell+1)}$ runs from $i-2 \ell$ to $i$, inclusive. This suggests that the following approximation should be good:

$$
\begin{aligned}
\mathbb{P}\left\{Y_{i}=y_{i} \mid Y_{<i} \text { and } X=x\right\} & \approx \mathbb{P}\left\{Y_{i}=y_{i} \mid Y_{i-2 \ell}^{(2 \ell+1)} \text { and } X_{i-3 \ell}^{(4 \ell+1)}\right\} \\
& =\frac{p_{2 \ell+1, \ell+1, \ell}\left(x_{i-3 \ell}^{(4 \ell+1)}, y_{i-2 \ell}^{(2 \ell+1)}\right)}{p_{2 \ell+1, \ell, \ell+1}\left(x_{i-3 \ell}^{(4 \ell+1)}, y_{i-2 \ell}^{(2 \ell)}\right)} .
\end{aligned}
$$

Since the full likelihood is a product across sites, we can then simply express an approximate full likelihood as a ratio of two composite likelihoods:

$$
\mathcal{L}(y \mid x) \approx \frac{A_{2 \ell+1, \ell+1, \ell}(x, y)}{A_{2 \ell+1, \ell, \ell+1}(x, y)} .
$$

See the next section for more discussion of the approximations.

\subsection{Choice of overhang}

The approximations in (4) and (6) hold if the overhang, $\ell$, is long enough. We present intuition about these formulas here, which provides a way to pick the overhang lengths $\ell$ and $r$; a precise statement is given in Appendix B. Suppose that each instantaneous change depends on positions at most $w$ sites away, and refer to $w$ as the "window width". Then, the mutation process at any particular site depends on the initial sequence at that site and within distance $w$ on either side... and the outcomes of any mutations that have occurred within that window (see Figure 2).

Therefore, the sequence at a particular site may directly affect the process at the neighboring $w$ sites on either side. If another change happens, say, at the end of this window, then the indirect effects of this 
change may extend out to $2 w$ sites. In this way, the influence of the sequence at each site extends outwards, as shown in Figure 2 - so if we take the overhangs long enough that this dependency is unlikely to extend to the base of the T-mer, the approximation will be good. As we show in Section B, if the overhangs are $\ell=r=k w$, then the error in approximation (4) is bounded by the probability that there are more than $k$ changes in a given window of length $w$. Concretely, sites further away than $k w$ only matter if there is a chain of at least $k$ intervening mutations - which is unlikely if $k$ is much larger than the expected number.

For instance, in the CpG model the window width is $w=1$ because the process only depends on neighboring sites; and if the sequence has evolved for time $t$ then we expect no more than a Poisson $(\lambda t)$ number of changes at each site. If $\lambda t=0.75\left(1-e^{-3 / 4}=52 \%\right.$ of the sequence will have changed!), then taking $\ell=r=3$ allows us to compute T-mer probabilities to within an error of 0.007 , since this is the probability that a Poisson with mean 0.75 will be larger than 3 . If $\lambda t=0.25$ we would do even better with $\ell=r=2$.

Appendix B makes this argument more precise. The argument for the full likelihood is more difficult, so at present our proposal above remains a conjecture. To see one difficulty, consider the TASEP model, with $x_{1}=y_{n}=1$, and all remaining positions equal to 0 . The full probability of this pair of sequences is very small, while the conditional probability of $Y_{n}=1$ given $X=10 \cdots 0$ and $Y_{<n}=0 \cdots 0$ is large since the 1 that initially began in the first position had to go somewhere. However, if we do not include the first position in the conditioning, this conditional probability is very different. Fortunately, such situations are rare, and the TASEP model provides the extreme case of dependency propagation. In practice, we recommend computing the likelihood with equation (6) using successively larger values of $\ell$ to check for convergence.

\section{Computation}

For inference, we need to compute the $|\mathcal{S}|^{n} \times|\mathcal{S}|^{m}$ matrix $F$ whose $(a, b)^{\text {th }}$ entry is $p_{n, \ell, r}(t, a, b)$, for various $t$ (and $m=n-\ell-r$ ). This matrix is a projection of the $|\mathcal{S}|^{n} \times|\mathcal{S}|^{n}$ matrix whose $(a, c)^{\text {th }}$ entry is

$$
p_{n}(a, c)=\left(e^{t G(n)}\right)_{a, c},
$$

where $G(n)$ is the sparse $|\mathcal{S}|^{n} \times|\mathcal{S}|^{n}$ matrix defined in (1). The "projection" we need just marginalizes over long (i.e., length $n$ ) patterns $c$ that match the shorter (i.e., length $m$ ) pattern $b$ as in (3): if we define the $n \times m$ matrix $U$ so that $U_{c b}=1$ if $c_{\ell}^{(m)}=b$ and $U_{c b}=0$ otherwise, then

$$
p_{n, \ell, r}(t, a, b)=\sum_{c \in \mathcal{S}^{n}}\left(e^{t G(n)}\right)_{a c} U_{c b}
$$

so in fact we don't need the entire matrix $e^{t G(n)}$, just the product of this matrix with each of the columns of $U$. Modern techniques in sparse matrix computation (e.g., Krylov methods) provide efficient ways to do this.

Using the $\mathrm{R}$ package expm [Goulet et al., 2017], this makes computation quite feasible: with four possible states and $\ell=2$ and $m=1$, so that $G(n)$ is a $1024 \times 1024$ matrix, computing $e^{t G(5)}$ takes 13 seconds, while computing $e^{t G(5)} U$ takes only 0.3 seconds. Increasing to $\ell=4$ and $m=1$ or $\ell=3$ and $m=2$ is still feasible, taking $e^{t G(8)} U$ in 42 and 47 seconds, respectively (and much longer for the whole matrix $e^{t G(8)}$ ).

Sparsity and updating $G$ We can also take advantage of the sparsity of $G$ to perform efficient computation of the likelihood under many sets of parameters. Let's assume that the T-mers only allow single-position changes. First note that in this case $G(n)$ has $(1+n(|\mathcal{S}|-1))|\mathcal{S}|^{n}$ nonzero entries, since each of the $|\mathcal{S}|^{n}$ sequences can change in $n$ places. This will determine how the computation scales with $n$ and $|\mathcal{S}|$, once we precompute a number of things. Let $g=\left(g_{1}, \ldots, g_{d}\right)$ be the nonzero entries of $G(n)$, in some fixed ordering; sparse matrix representations of $G(n)$ store only $g$ along with information about the rows and columns these are found in. Each $g_{i}$ is a linear combination of mutation rates $\mu_{j}$, multiplied by a function $(\phi)$ of a linear combination of energy coefficients $e_{j}$, say $g_{i}=\sum_{j} A_{i j} \mu_{j} \phi\left(\sum_{k} B_{i k} e_{k}\right)$, so by precomputing the matrices $A$ 
and $B$ we can update $G(n)$ with new parameter values using only two matrix multiplications and evaluation of $\phi()$ over a vector. This remains efficient to perform in interpreted languages such as $\mathrm{R}$, since each step is carried out by lower-level compiled code (e.g., optimized linear algebra libraries).

\section{Phylogenetic inference}

In phylogenetic applications, rather than "before" and "after" observations, we get two (or more) observations evolved from a common root. In the simplest case of two tips we have two processes $X$ and $Y$, with identical starting states $X(0)=Y(0)$, observed only at times $t_{X}$ and $t_{Y}$ respectively. At first, one might think to compute probabilities with a "long" sequence at the root and "short" sequences at every tip. However, this does not work out, since the root is unobserved and short sequences at every tip give us no information about what the root sequence in the overhangs might be.

Instead, we will compute the probability that we see (long) pattern $x$ in $X\left(t_{X}\right)$ juxtaposed with (short) pattern $y$ in $Y\left(t_{Y}\right)$, summing over possible (long) patterns at the root. Concretely: pick a random location $I$ in the sequence and let $X_{I-\ell}^{(n)}(0)=\rho_{I}$ be the (long) pattern seen there at the root. Write $\pi(z)$ for the frequency of a given pattern $z$ seen there, i.e., $\mathbb{P}\left\{\rho_{I}=z\right\}=\pi(z)$. The probability that we see $x$ and $y$ at the random location $I$ is

$$
\mathbb{P}\left\{X_{I-\ell}^{(n)}\left(t_{X}\right)=x \text { and } Y_{I}^{(m)}\left(t_{Y}\right)=y\right\}=\sum_{z \in \mathcal{S}^{n}} \pi(z) p_{n, 0,0}\left(t_{X}, z, x\right) p_{n, \ell, r}\left(t_{Y}, z, y\right) .
$$

Denote this quantity $p_{n, \ell, r}(x, y)$. This can be computed without much more effort than the simpler case above, given the frequencies at the root, as described in greater generality below. These calculations can be done using the same sparse matrix methods as above. For instance, to compute

$$
\sum_{z \in \mathcal{S}^{n}} \pi(z) p_{n, 0,0}\left(t_{X}, z, x\right) p_{n, \ell, r}\left(t_{Y}, z, y\right)=\sum_{z \in \mathcal{S}^{n}}\left(e^{t_{X} G(n)}\right)_{z x} \pi(z) \sum_{w \in \mathcal{S}^{n}}\left(e^{t_{Y} G(n)}\right)_{z w} U_{w y}
$$

we can first compute $\sum_{w}\left(e^{t_{Y} G(n)}\right)_{z w} U_{w y}$ as above, multiply rows by $\pi(z)$, and then matrix multiply by the transpose of $e^{t_{X} G(n)}$.

We might approximate the full likelihood as follows, after choosing $n, \ell$, and $r$ appropriately (see above). For the sake of notational simplicity, assume that we have a few positions of $X$ observed before the start and after then end of the sequence (so that, e.g., we can refer to $x_{n+1}, \ldots, x_{n+r}$ ).

$$
\begin{aligned}
\mathcal{L}(x, y) & =\prod_{i=1}^{L} \mathbb{P}\left\{X_{i+r}=x_{i+r}, Y_{i}=y_{i} \mid X_{<i+r}=x_{<i+r}, Y_{<i}=y_{<i}\right\} \\
& \approx \prod_{i=1}^{L} \mathbb{P}\left\{X_{i+r}=x_{i+r}, Y_{i}=y_{i} \mid X_{i-\ell}^{(n-1)}=x_{i-\ell}^{(n-1)}, Y_{i}^{(m-1)}=y_{i}^{(m-1)}\right\} \\
& =\prod_{i=1}^{L} \frac{p_{n, \ell, r}\left(x_{i-\ell}^{(n)}, y_{i}^{(m)}\right)}{p_{n-1, \ell, r}\left(x_{i-\ell}^{(n-1)}, y_{i}^{(m-1)}\right)}
\end{aligned}
$$

We expect this to be a good approximation to the true likelihood when the same conditions above are met, taking $t$ to the be the largest distance between two taxa in the tree.

\subsection{Phylogenetic pruning}

The general case is a modification of Felsenstein's "pruning" algorithm [Felsenstein, 1981].

To compute the likelihood on a more complicated tree, consider the following, with the three-taxon tree of Figure 3 as an example, where we are counting occurrences of (long) $n$-tuples in taxon $X$, and (shorter) 


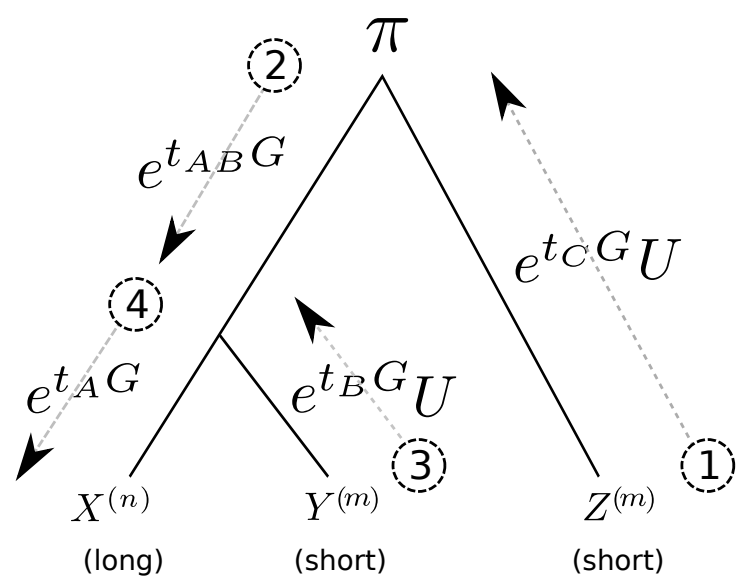

Figure 3: A depiction of the four steps in computing the likelihood of finding particular "short" subsequences at $Y$ and $Z$ given the "long" subsequence at $X$ : see text for details.

$m$-tuples in taxa $Y$ and $Z$. The likelihood in this example is found by summing over the root state (the summation over $u$ ) and the interior node $(v)$ :

$$
\begin{aligned}
& \mathbb{P}\left\{X_{I-\ell}^{n}=x \text { and } Y_{I}^{\ell}=y \text { and } Z_{I}^{\ell}=z\right\} \\
& \quad=\sum_{u \in \mathcal{S}^{n}} \pi(u) p_{n, \ell, r}\left(t_{C}, u, z\right) \sum_{v \in \mathcal{S}^{n}} p_{n, 0,0}\left(t_{A B}, u, v\right) p_{n, 0,0}\left(t_{A}, v, x\right) p_{n, \ell, r}\left(t_{B}, v, y\right)
\end{aligned}
$$

The steps for computing this are then:

1. Compute $M_{1}(u, z)=p_{m, \ell, r}\left(t_{C}, u, z\right)\left(\mathrm{a}|\mathcal{S}|^{n} \times|\mathcal{S}|^{m}\right.$ matrix $)$

2. Compute $M_{2}(v, z)=\sum_{u \in \mathcal{S}^{n}} p_{n, 0,0}\left(t_{A B}, u, v\right) \pi(u) M_{1}(u, z)$ (still a $|\mathcal{S}|^{n} \times|\mathcal{S}|^{m}$ matrix)

3. Compute $M_{3}(v, y)=p_{m, \ell, r}\left(t_{B}, v, y\right)\left(\mathrm{a}|\mathcal{S}|^{n} \times|\mathcal{S}|^{m}\right.$ matrix $)$

4. Compute $M_{4}(x,(y, z))=\sum_{v \in \mathcal{S}^{n}} p_{n, 0,0}\left(t_{A}, v, x\right) M_{2}(v, z) M_{3}(v, y)\left(\right.$ stored as a $|\mathcal{S}|^{n} \times|\mathcal{S}|^{2 m}$ matrix).

Then $M_{4}$ gives all the likelihoods. This is depicted in the figure, writing $\left(e^{t G}\right)_{a b}$ for the matrix $p_{n, 0,0}(t, a, b)$, and $U$ for the projection matrix that gives $p_{m, \ell, r}(t, a, b)=\left(e^{t G} U\right)_{a b}$.

In total there are $|\mathcal{S}|^{3 m+2 \ell}$ possible data combinations. However, we can reduce dimensionality by only computing probabilities for certain (more common) patterns: for instance, one could compute the likelihood for situations where $y$ and $z$ agree (but may differ from $x$ by replacing step (4) with:

$$
4^{\prime} \text {. Compute } M_{4}(x, w)=\sum_{v \in \mathcal{S}^{n}} p_{n, 0,0}\left(t_{A}, v, x\right) M_{3}(v, w) M_{4}(v, w)\left(\text { stored as a }|\mathcal{S}|^{n} \times|\mathcal{S}|^{m}\right. \text { matrix). }
$$

On a three-taxon tree it is not clear that this is desirable, but on larger trees it seems likely that we'd want to restrict to combinations with most sites agreeing across the tree. (Note, however, that one should not first look at the data, observe which combinations $(y, z)$ were most common, and then restrict to only those!)

The algorithm for a general tree is written out in Appendix C.

\subsection{Model fit and model selection}

Fitting a model to nucleotide data will likely require iterative modification of the model. The difference between observed and expected T-mer counts provide a good assessment of how and whether a model can be improved. These residual counts are usually most usefully computed for T-mers shorter than those used 
to fit the model, because the smaller total number of possible T-mers makes it easier to identify the shared patterns. For each T-mer $(x, y)$, write $O_{x, y}$ for the number of this T-mer observed in the data, and $E_{x, y}$ for the number expected under the model. (The expected number is the number of occurrences of $x$ in the initial sequence multiplied by the probability $p_{n, \ell, r}(t ; x, y)$, where $n$ is the length of $x$, and $\ell, r$ are the appropriate overhangs.) If the T-mers were nonoverlapping, this would form a standard contingency table, and standard practice would be to divide the residuals by the square root of the expected counts and compare these to a standard Normal distribution. However, there are are $n$ possible such tables - so, we compute normalized residuals as

$$
Z_{x, y}=\frac{O_{x, y}-E_{x, y}}{\sqrt{n E_{x, y}}}
$$

which is equivalent to averaging the $z$-scores of the $n$ possible tables of nonoverlapping counts. These can then be compared to a standard Normal distribution.

\section{Software}

The methods described here are implemented in R and freely available at https://github.com/petrelharp/ context. The core implementation of T-mer counting and likelihood evaluation is provided in an $\mathrm{R}$ package, contextual, and is supplemented by (documented) code to simulate from context-dependent models and perform maximum likelihood or Bayesian inference.

\section{Results}

\subsection{Stepwise model selection}

We benchmarked the performance of stepwise model selection: (a) fit a simple model, (b) choose new motifs to add based on statistically significant residuals, (c) repeat until no residuals are statistically significant. To test this method, we simulated a single sequence of $10^{6}$ nucleotides for one unit of time under the model given in Table 1, obtained by embellishing the basic CpG hypermutability model with the longer mutational motif identified by Harris [2015]. The resulting sequences differed at $28.5 \%$ of the sites.

To begin, we fit an unconstrained non-context-dependent model of single-nucleotide substitution using a constrained quasi-Newton method (optim(.., method='L-BFGS-B') in R). Recall that for inference, we next need to pick a T-mer shape with which to compute likelihoods, and the shape of this T-mer is only determined by the shape of dependencies in the generative model in that it determines the minimum base and overhang lengths. We use $(5,1,1)$ T-mers: three sites at the base with an overhang of one on each side. Since this initial model has no context-dependence, we could do the first step of model fitting using $(1,0,0)$ T-mers (i.e., single positions), but for simplicity we use the same T-mer shape for all steps.

After fitting the model, we can look at residuals using any T-mer shape we like. At this first step, we computed the $(2,1,0)$ and $(2,0,1)$ T-mer residuals, shown in Table 2. The largest residuals are an excess of $\mathrm{C} \rightarrow \mathrm{T}$ mutations in $\mathrm{CG}$ dinucleotides, and the reverse-complement pattern ( $z$ scores of 88). Complementing this are deficits of $\mathrm{C} \rightarrow \mathrm{T}$ mutations in other contexts, since the $\mathrm{C} \rightarrow \mathrm{T}$ mutation rate has been fit to be an average across contexts. This clearly calls for addition of the CG $\rightarrow$ TG mutation motif (and reverse complement), which was used in simulating the data.

After adding these patterns into the model and re-fitting, we searched for evidence of additional processes happening in the data. To do so, we calculated length 2 residuals and $(3,1,1)$ T-mer residuals. Although some of the length 2 residuals had large $z$-scores, none were as large as those seen in $(3,1,1)$ T-mer residuals, which are shown in Table 3. These show an excess of TCC $\rightarrow$ TTC and AGG $\rightarrow$ AAG changes, which were indeed the motifs used when simulating the data. After adding these in, no further $(3,1,1)$ T-mer residuals achieved statistical significance after computing $p$-values with the Gaussian CDF and applying a Bonferroni correction. The final parameter values are shown in Table 1; parameter estimates differ from the truth by less than $3 \%$ (mostly within 1\%). 


\begin{tabular}{clllll}
\hline $\mathrm{x}$ & $\rightarrow$ & $\mathrm{y}$ & at rate & truth & MLE \\
\hline $\mathrm{A}$ & $\rightarrow$ & $\mathrm{T}$ & at rate & 0.10 & 0.0996 \\
$\mathrm{~T}$ & $\rightarrow$ & $\mathrm{A}$ & at rate & 0.10 & 0.0982 \\
$\mathrm{C}$ & $\rightarrow$ & $\mathrm{G}$ & at rate & 0.15 & 0.1505 \\
$\mathrm{G}$ & $\rightarrow$ & $\mathrm{C}$ & at rate & 0.15 & 0.1497 \\
$\mathrm{~A}$ & $\rightarrow$ & $\mathrm{C}$ & at rate & 0.08 & 0.0784 \\
$\mathrm{~T}$ & $\rightarrow$ & $\mathrm{G}$ & at rate & 0.08 & 0.0792 \\
$\mathrm{~A}$ & $\rightarrow$ & $\mathrm{G}$ & at rate & 0.08 & 0.0798 \\
$\mathrm{~T}$ & $\rightarrow$ & $\mathrm{C}$ & at rate & 0.08 & 0.0795 \\
$\mathrm{C}$ & $\rightarrow$ & $\mathrm{A}$ & at rate & 0.12 & 0.1202 \\
$\mathrm{G}$ & $\rightarrow$ & $\mathrm{T}$ & at rate & 0.12 & 0.1196 \\
$\mathrm{C}$ & $\rightarrow$ & $\mathrm{T}$ & at rate & 0.12 & 0.1198 \\
$\mathrm{G}$ & $\rightarrow$ & $\mathrm{A}$ & at rate & 0.12 & 0.1193 \\
$\mathrm{CG}$ & $\rightarrow$ & $\mathrm{TG}$ & at rate & 0.40 & 0.3978 \\
$\mathrm{CG}$ & $\rightarrow$ & $\mathrm{CA}$ & at rate & 0.40 & 0.3978 \\
$\mathrm{TCC}$ & $\rightarrow$ & $\mathrm{TTC}$ & at rate & 0.10 & 0.1033 \\
$\mathrm{AGG}$ & $\rightarrow$ & $\mathrm{AAG}$ & at rate & 0.10 & 0.1033 \\
\hline
\end{tabular}

Table 1: True parameter values, and MLE estimates obtained from iterative fitting as described in the text to a $10^{6}$ bp sequence evolved to have $28.5 \%$ sequence divergence with the above mutational motifs. The transitions $\mathrm{CG} \rightarrow \mathrm{TG}$ and $\mathrm{CG} \rightarrow \mathrm{CA}$ were constrained during fitting to be equal (DNA strand symmetry), as were the transitions TCC $\rightarrow$ TTC and AGG $\rightarrow$ AAG.

\subsection{Bayesian inference}

The usage of fast sparse matrix methods described above make likelihood computation fast enough to use a Metropolis-Hastings-based Markov chain Monte Carlo method to sample from the posterior distribution on parameters. To test this, we evolved $10^{6}$ sites from the Ising model (described above) with $\lambda=0.5, \beta=1.0$, and $\gamma=0.5$ for 1 unit of time, resulting in around 460,000 possible mutations and differences at around 186,000 sites between initial and final sequence. We then placed an Exponential prior with mean 1 on both transitions $+\rightarrow-$ and $-\rightarrow+$ (without constraining these to be equal) and half-Gaussian priors with mean 0 and scale 3 on both $\beta$ and $\gamma$, counted $(5,3,1)$ T-mers, and used the mcmc package [Geyer and Johnson, 2017 to run a random walk sampler for 10,000 steps (judged sufficient by examination of diagnostic plots). We repeated this procedure separately on 61 independently simulated sequences, and show the resulting credible intervals in Figure 4.

Posterior medians were within half a percent of the truth for $+\rightarrow-$ and $-\rightarrow+$ (corresponding to $\lambda$ ) and the temperature $(\beta)$, but showed around $2 \%$ error for the magnetic field $\gamma$. As seen in Table 4 , credible intervals were somewhat too narrow for shorter T-mers, but $(6,2,2)$ T-mers were long enough with sufficient overhang to produce well-calibrated posteriors.

\subsection{Hominid mutation spectrum}

We also applied the method to human-chimpanzee divergence data, restricting the comparison to putative regulatory sequences, avoiding the additional constraints of coding sequences. To do this, we extracted regions in the human (hg38)-chimpanzee (panTro4) alignment according to four types of regulatory feature in the Ensembl Regulatory Build [release 81 Zerbino et al., 2015]: "CTCF binding site", "enhancer", "open chromatin region", and "promoter flanking region". These were furthermore divided into regions that either did or did not overlap a transcription start or end site of a known gene from the hg38 Annotations Database [Kent et al., 2002] and filtered for length: aligned regions below either 3000bp (promoter flanking regions) or 1000bp (all other types). We then counted T-mers of appropriate length in each of the eight resulting sets of aligned regions, omitting any T-mers that included gaps or repeat-masked positions, and fit models 


\begin{tabular}{ccrrrr}
$x$ & $y$ & observed & expected & residual & $Z$ \\
\hline CG & $\mathrm{C}_{-}$ & 100410 & 120303 & -19892.50 & -40.55 \\
$\mathrm{CA}$ & $\mathrm{T}_{-}$ & 19884 & 28350 & -8466.43 & -35.55 \\
$\mathrm{CT}$ & $\mathrm{T}_{-}$ & 19998 & 28061 & -8063.46 & -34.03 \\
$\mathrm{CC}$ & $\mathrm{T}_{-}$ & 23673 & 28177 & -4503.96 & -18.97 \\
$\mathrm{GG}$ & $\mathrm{C}_{-}$ & 17742 & 19418 & -1675.92 & -8.50 \\
$\mathrm{CG}$ & $\mathrm{G}_{-}$ & 18450 & 19478 & -1028.34 & -5.21 \\
$\mathrm{AG}$ & $\mathrm{C}_{-}$ & 10968 & 11760 & -791.73 & -5.16 \\
\hline $\mathrm{GT}$ & $\mathrm{C}_{-}$ & 20490 & 19507 & 983.44 & 4.97 \\
$\mathrm{GG}$ & $\mathrm{T}_{-}$ & 20929 & 19278 & 1650.83 & 8.40 \\
$\mathrm{CC}$ & $\mathrm{C}_{-}$ & 124149 & 119880 & 4268.52 & 8.71 \\
$\mathrm{GG}$ & $\mathrm{A}$ & 30483 & 28288 & 2195.34 & 9.22 \\
$\mathrm{CT}$ & $\mathrm{C}_{-}$ & 126957 & 119389 & 7567.92 & 15.48 \\
$\mathrm{CA}$ & $\mathrm{C}-$ & 128673 & 120619 & 8054.46 & 16.39 \\
$\mathrm{CG}$ & $\mathrm{T}-$ & 49311 & 28276 & 21034.85 & 88.45 \\
\hline \hline $\mathrm{CG}$ & $-\mathrm{G}$ & 100803 & 120541 & -19737.80 & -40.19 \\
$\mathrm{TG}$ & $-\mathrm{A}$ & 20040 & 28179 & -8139.45 & -34.28 \\
$\mathrm{GG}$ & $-\mathrm{A}$ & 20985 & 28288 & -7302.96 & -30.70 \\
$\mathrm{AG}$ & $-\mathrm{A}$ & 22929 & 28399 & -5469.73 & -22.95 \\
$\mathrm{CC}$ & $-\mathrm{G}$ & 17805 & 19410 & -1605.01 & -8.14 \\
$\mathrm{CT}$ & $-\mathrm{G}$ & 10470 & 11684 & -1214.31 & -7.94 \\
$\mathrm{CA}$ & $-\mathrm{G}$ & 10839 & 11882 & -1043.39 & -6.76 \\
\hline $\mathrm{TC}$ & $-\mathrm{G}$ & 20691 & 19509 & 1182.40 & 5.98 \\
$\mathrm{CC}$ & $-\mathrm{A}$ & 20640 & 19160 & 1480.44 & 7.56 \\
$\mathrm{TC}$ & $-\mathrm{T}$ & 30309 & 28320 & 1988.91 & 8.35 \\
$\mathrm{AG}$ & $-\mathrm{G}$ & 127191 & 121450 & 5740.76 & 11.64 \\
$\mathrm{GG}$ & $-\mathrm{G}$ & 127946 & 120977 & 6969.46 & 14.16 \\
$\mathrm{TG}$ & $-\mathrm{G}$ & 127539 & 120512 & 7026.52 & 14.31 \\
$\mathrm{CG}$ & $-\mathrm{A}$ & 49098 & 28186 & 20911.93 & 88.07 \\
\hline & & & & &
\end{tabular}

Table 2: Largest residuals of shape $(2,1,0)$ and $(2,0,1)$ after fitting a model with only "basic" (singlenucleotide) mutations to sequence simulated under the model of Table 1, that also includes CpG hypermutability as well as TCC $\rightarrow$ TTC and AGG $\rightarrow$ AAG mutations. Note that in the table, the underscore means "any nucleotide". 


\begin{tabular}{|c|c|c|c|c|c|}
\hline$x$ & $y$ & observed & expected & residual & $\bar{Z}$ \\
\hline AGG & $\mathrm{G}_{-}$ & 30024 & 32167 & -2143.48 & -6.90 \\
\hline TCC & $\mathrm{C}_{-}$ & 29910 & 31748 & -1838.13 & -5.95 \\
\hline GGT & $\mathrm{A}_{-}$ & 5064 & 5549 & -484.61 & -3.75 \\
\hline TGC & $\mathrm{A}_{-}$ & 4830 & 5172 & -342.42 & -2.74 \\
\hline GGA & $\mathrm{A}_{-}$ & 5115 & 5462 & -346.97 & -2.71 \\
\hline CTT & ${ }_{-} G_{-}$ & 2472 & 2717 & -244.56 & -2.70 \\
\hline GCT & $-T_{-}$ & 4863 & 5192 & -328.56 & -2.63 \\
\hline ATG & $\mathrm{G}_{-}$ & 3225 & 3028 & 196.78 & 2.06 \\
\hline ACG & $\mathrm{A}_{-}$ & 4830 & 4588 & 242.29 & 2.06 \\
\hline TGT & ${ }_{-} \mathrm{C}_{-}$ & 5364 & 5105 & 258.74 & 2.09 \\
\hline GAG & $\mathrm{G}_{-}$ & 3285 & 3055 & 229.78 & 2.40 \\
\hline TTG & $\mathrm{C}_{-}$ & 2973 & 2752 & 220.59 & 2.42 \\
\hline TCT & $\mathrm{G}_{-}$ & 5421 & 5056 & 364.54 & 2.95 \\
\hline TCC & $\mathrm{T}_{-}$ & 7590 & 5500 & 2089.79 & 16.26 \\
\hline AGG & $\mathrm{A}_{-}$ & 7566 & 5263 & 2302.74 & 18.32 \\
\hline
\end{tabular}

Table 3: Largest residuals of shape $(3,1,1)$ after fitting a model with "basic" (single-nucleotide) and CpG mutations to sequence simulated under the model of Table 1, that also includes TCC $\rightarrow$ TTC and AGG $\rightarrow$ AAG mutations. Note that in the table, the underscore means "any nucleotide".

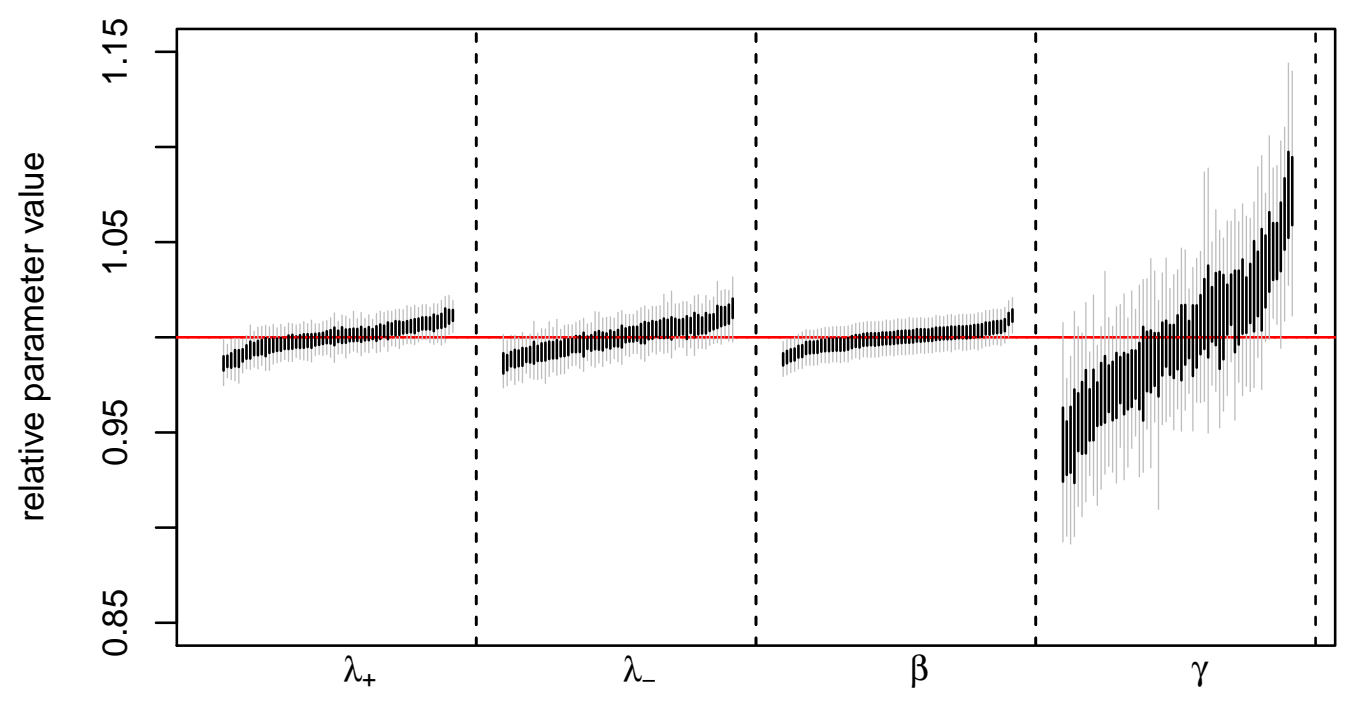

Figure 4: Credible intervals for the four parameters of the dynamic Ising model, calculated using $(6,2,2)$ T-mers, from $61 \mathrm{MCMC}$ runs on independently simulated sequences of $10^{6}$ bases differing at around $18.6 \%$ of the sites. Dark bars show the $50 \%$ credible intervals, and lighter grey bars the $95 \%$ credible intervals. Values shown are relative parameters, obtained by dividing by the true value. 


\begin{tabular}{rrrr}
\hline & $(4,1,1)$ & $(5,1,1)$ & $(6,2,2)$ \\
\hline$\lambda_{-}$ & 0.61 & 0.63 & 0.96 \\
$\lambda_{+}$ & 0.87 & 0.86 & 0.85 \\
$\beta$ & 0.76 & 0.81 & 0.94 \\
$\gamma$ & 0.69 & 0.76 & 0.85 \\
\hline
\end{tabular}

Table 4: Posterior coverage of the four parameters of the dynamic Ising model at four different T-mer sizes. Shown are the proportion of runs in which the true value fell within the $95 \%$ credible interval, for T-mers of length 4, 5, and 6 with overhangs of length 1, 1, and 2 respectively. The total number of runs was 61 for $(6,2,2) \mathrm{T}$-mers and 121 for the others.

separately to each of the eight data sets.

We constructed models from three, nested, sets of mutation motifs. All models are strand-symmetric.

(basic) Single-base changes, with separate rates for transitions $\left(\mu_{v}\right), \mathrm{A} \leftrightarrow \mathrm{T}\left(\mu_{A T}\right), \mathrm{C} \leftrightarrow \mathrm{G}\left(\mu_{C G}\right)$, and other transversions $\left(\mu_{r}\right)$ :

$$
\begin{aligned}
& \mathrm{T} \rightarrow \mathrm{A} \text { at rate } \mu_{A T} \mid \mathrm{A} \rightarrow \mathrm{C} \text { at rate } \mu_{r} \mid \mathrm{A} \rightarrow \mathrm{G} \text { at rate } \mu_{v} \\
& \mathrm{~A} \rightarrow \mathrm{T} \text { at rate } \mu_{A T} \quad \mathrm{C} \rightarrow \mathrm{A} \text { at rate } \mu_{r} \mathrm{G} \rightarrow \mathrm{A} \text { at rate } \mu_{v} \\
& \mathrm{C} \rightarrow \mathrm{G} \text { at rate } \mu_{C G} \quad \mathrm{G} \rightarrow \mathrm{T} \text { at rate } \mu_{r} \mathrm{~T} \rightarrow \mathrm{C} \text { at rate } \mu_{v} \\
& \mathrm{G} \rightarrow \mathrm{C} \text { at rate } \mu_{C G} \mathrm{~T} \rightarrow \mathrm{G} \text { at rate } \mu_{r} \mathrm{C} \rightarrow \mathrm{T} \text { at rate } \mu_{v}
\end{aligned}
$$

(CpG) In addition to the above, an additional $\mathrm{CpG}$ rate:

$$
\begin{aligned}
& \mathrm{CG} \rightarrow \mathrm{TG} \text { at rate } \nu_{C p G} \\
& \mathrm{CG} \rightarrow \mathrm{CA} \text { at rate } \nu_{C p G}
\end{aligned}
$$

(repair) In addition to the above, we included a collection of mutation motifs associated with known DNA lesion/repair pathways curated from the literature [reviewed in Sale et al., 2012, Goodman and Woodgate, 2013, Roberts and Gordenin, 2014, Cobey et al., 2015, Goodman, 2002]: deamination due to repair of UV-induced pyramidine dimers [ $\mu_{U V}$, Sale et al., 2012, Sinha and Hader, 2002]; repair of guanine adducts at $\mathrm{CpG}$ sites Pfeifer $\left[\mu_{g}, 2006\right]$; deamination by AID [ $\mu_{\text {AID }}$, Teng and Papavasiliou, 2007, Kasar

\begin{tabular}{|c|c|c|c|c|c|c|c|c|}
\hline $\mathrm{CG}$ & $\rightarrow \mathrm{CT}$ & at rate & $\mu_{g}$ & $\mathrm{AA}$ & $\rightarrow$ & $\mathrm{AG}$ & at rate & $\mu_{\iota 1}$ \\
\hline $\mathrm{CG}$ & $\rightarrow \mathrm{AG}$ & at rate & $\mu_{g}$ & TT & $\rightarrow$ & $\mathrm{CT}$ & at rate & $\mu_{\iota 1}$ \\
\hline$\Gamma \mathrm{C}$ & $\rightarrow \mathrm{TT}$ & at rate & $\mu_{U V}$ & $\mathrm{AA}$ & $\rightarrow$ & GT & at rate & $\mu_{\iota 2}$ \\
\hline$A$ & $\rightarrow \mathrm{AA}$ & at rate & $\mu_{U V}$ & $\mathrm{TT}$ & $\rightarrow$ & $\mathrm{AC}$ & at rate & $\mu_{\iota 2}$ \\
\hline $\mathrm{C}$ & $\rightarrow \mathrm{CT}$ & at rate & $\mu_{U V}$ & TCW & $\rightarrow$ & TTW & ate & $\mu_{\text {APOBEC }}$ \\
\hline G & $\rightarrow \mathrm{AG}$ & at rate & $\mu_{U V}$ & TCW & $\rightarrow$ & TGW & at rate & $\mu_{\mathrm{APOBEC}}$ \\
\hline $\mathrm{CC}$ & $\rightarrow \mathrm{TT}$ & at rate & $\mu_{U V d}$ & SGA & $\rightarrow$ & SAA & at rate & $\mu_{\mathrm{APOBEC}}$ \\
\hline $\mathrm{G}$ & $\rightarrow \mathrm{AA}$ & at rate & $\mu_{U V d}$ & SGA & $\rightarrow$ & SCA & at rate & $\mu_{\mathrm{APOBEC}}$ \\
\hline & $\rightarrow \mathrm{TG}$ & at rate & $\mu_{\eta}$ & WRCY & $\rightarrow$ & WRTY & at rate & $\mu_{\mathrm{AID}}$ \\
\hline$A$ & $\rightarrow \mathrm{CA}$ & at rate & $\mu_{\eta}$ & RGYS & $\rightarrow$ & RAYS & at rate & $\mu_{\mathrm{AID}}$ \\
\hline
\end{tabular}
et al., 2015]; deamination by members of the APOBEC family [ $\mu_{\mathrm{APOBEC}}$, Alexandrov et al., 2013a]; and repair by error-prone polymerases pol $\eta\left[\mu_{\eta}\right.$, Alexandrov et al., 2013a]; and pol $\iota\left[\mu_{\iota}\right.$, Roberts and Gordenin, 2014, Maul et al., 2016]. These are denoted here using ambiguity codes R: A or G, Y: C or T, W: A or T, and S: C or G; so for instance "TCW $\rightarrow$ TTW" means that both TCA $\rightarrow$ TTA and $\mathrm{TCT} \rightarrow \mathrm{TTT}$ share the same mutation rate.

The mutational motifs included above are far from complete - context-specific affinities and error rates in vivo are not known for most DNA repair pathways - but neither do they exhaust the hypotheses available in the literature. For instance, many enzymes act more than once locally when they act, such as AID [Senavirathne et al., 2015] or error-prone polymerases involved in repair [Maul et al., 2016]. 


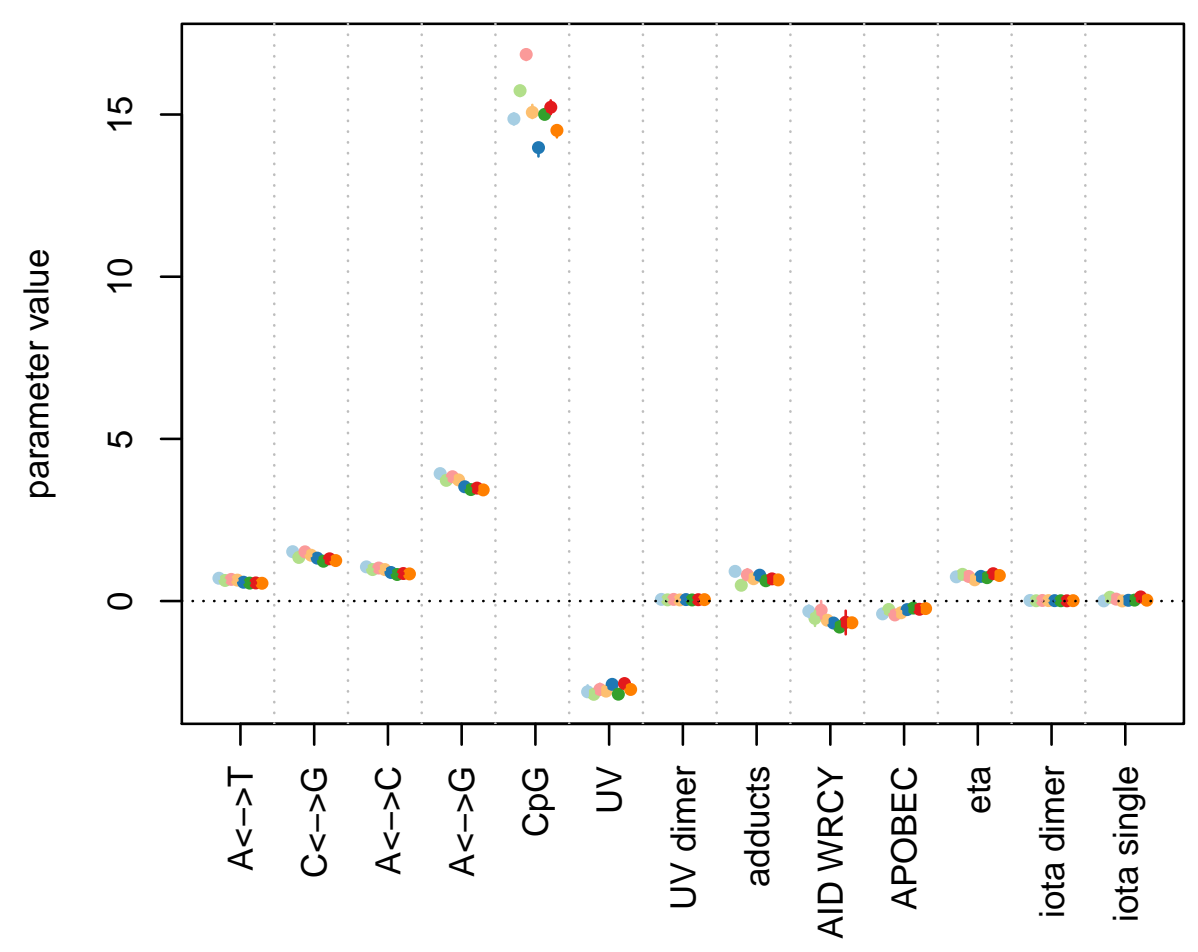

- nongene : CTCF_binding_site - nongene : enhancer

nongene : open_chromatin_region

nongene : promoter_flanking_region

- gene : enhancer

- gene : open chromatin region

- gene : promoter_flanking_region

Figure 5: Estimated average mutation rates for the motifs in the "Hominid data" section since the common ancestor of humans and chimpanzee. Rates are scaled so that the mean time to common ancestor is 0.001 units - if we take this to be 6 million years [Scally et al., 2012, Langergraber et al., 2012] then rates are in units of mutations per 6,000 years. Shown are posterior medians (points) and 95\% credible intervals (lines, which are quite small in all cases). Figure S2 shows the smaller rates in more detail, and Figure S3 shows the rates between categories relative to each other.

Nevertheless, we find significant effects for a number of biochemically-informed mutation features (Figure 5). Specifically, we find strong CpG and UV damage effects [Harris, 2015, also seen by]. It may seem tenuous to look for mutational signatures associated with cancer in the germline, or surprising that UV damage could play a significant role, but it is reasonable that shared repair machinery might induce similar context-dependent errors. Other likely sources of error in the germline include the effects of recombination [Arbeithuber et al., 2015, Myers et al., 2010].

\section{Discussion}

This paper presents a tractable method for doing statistical inference using observations of a particle model in which probabilities of change are affected by nearby sites. Such models appear in many fields, for instance, as the dynamic counterparts to Markov random fields or spin glass models, and models of DNA sequence evolution.

The methods described here provide a way to efficiently approximate full likelihoods, which in many circumstances provide the optimal basis for inference [Neyman and Pearson, 1933], fast enough to allow Bayesian inference from Monte Carlo sampling. The method is fast thanks to its dependence on only local neighborhoods (so the time does not scale with sequence length once T-mer abundances are counted) and 
its reliance on sparse matrices whose structure can be precomputed and cached. It avoids approximations made by previous methods thanks to the use of T-mers and explicit quantification of the necessary length of the "arms" of the T-mers. We first discuss possible uses of the method, followed by challenges (and possible solutions).

Other applications One class of applications stems from inference of process - where the numerical values of various mutation rates are directly of interest. For instance, $\mathrm{CpG}$ hypermutability depends on the CGpair being methylated, an epigenetic modification that has important consequences for gene regulation; therefore, inference of $\mathrm{CpG}$ mutation rates in ancient taxa provides an indirect estimate of methylation status in extinct organisms. Similarly, many mutational motifs are thought to be caused by particular enzymes; inference of relative rates of these motifs may provide clues as to the activity and evolution of those enzymes. Furthermore, iterative model building by examining residual motif counts could provide a powerful way to discover unknown processes whose action had been obscured by other sources of noise.

In other applications, reconstruction of an unknown sequence may be of interest. For instance, training data might be used to estimate parameters of a noisy transmission line, facilitating the later reconstruction of noisily transmitted signals. Alternatively, multiple observations of independently transmitted copies of a single sequence (e.g., down a phylogenetic tree) might be used to estimate the process, and from this infer the original (ancestral) sequence (or sample from its posterior distribution).

Limitations The model presented assumes that the same process occurs at every location along the sequence, and that the length of the sequence does not change (no insertions or deletions). Neither of these assumptions are likely to be true for any data set of DNA sequence; however, both assumptions are also commonly made in phylogenetics. Some relaxation of the assumptions is possible - for instance, we can easily allow heterogeneity between sites by scaling rates by an overall gamma-distributed random factor [Yang, 1994]. Insertions and deletions are problematic, however.

Furthermore, we have not been able to put quantitative bounds on how well we can approximate the full likelihood with a given T-mer shape. One reason this is difficult is that there are special cases of initial and final sequence for which the likelihood is not approximable by looking only at T-mer counts; however, we believe such situations to be rare, so that the full likelihood can be well approximated on a set of outcomes of high probability. The TASEP model represents in some ways the worst-case of dependency propagation, and has an analytic solution [Schütz, 1997]. Further theoretical work in this direction would be welcome. In practice, the approximation may be checked by computing the likelihood using successively longer T-mers, and checking for convergence as the length (of both base and overhang) increase.

Computation The main factor that determines computation time is the lengths of the T-mers used for inference, since all computations scale with the number of possible patterns at the long end of the T-mer. Although the model is efficient enough to deal with fairly long T-mers, this exponential growth puts hard constraints on the application of the method. The length of T-mers required to accurately compute the likelihood in most models grows at most linearly with the mean density of substitution between the initial and final sequence. This does not in practice greatly increase the length of required T-mer since substitution densities approaching $100 \%$ are unlikely to be usable in practice especially if how the sequences align with each other is unknown. A more serious determining factor of T-mer length is the degree of dependency in the model itself. For instance, inserting the 13-base pair motif selected against by meiotic drive in primates [Myers et al., 2010] would require T-mers of width at least 25 , and hence vectors of length $4^{25} \approx 1.13 \times 10^{15}$.

Modeling Careful building of complex models in this framework may take care, but no more than in any other class of models (e.g., multivariate linear regression). It is easy to specify nonidentifiable models - for instance, all 2 mer mutation motifs include the 1mer motifs. The large number of possible mutation motifs also makes multiple testing an issue; an unguided approach might include all possible motifs but place a shrinkage prior on their values [Bhadra et al., 2015]. The converse approach of adding motifs that are overrepresented in the residuals may be quicker, but entails some degree of arbitrariness in choosing the 
motifs to add (and in which order). Finally, inference on trees may be difficult unless the root placement and distribution are strongly constrained: otherwise, initial investigations suggest the likelihood surface is often strongly ridged - for instance, increasing the abundance of $G$ nucleotides at the root while also increasing the mutation rate away from $G$ can achieve roughly the same result. However, in phylogenetic applications outgroups can be used to constrain these quantities.

In the genomic context, if we knew the specific mutational spectrum of each possible repair or copying pathways, then this framework could be used to rigorously estimate their average usages and impacts. Despite substantial progress over the past two decades [Goodman and Woodgate, 2013, Sale et al., 2012], this is still far from known. A promising alternative would be to use the distinct signatures obtained by dimension reduction techniques as in Alexandrov et al. [2013b], [Mathieson and Reich, 2017] or [Shiraishi et al., 2015] as a proxy for unknown but distinct pathways.

\subsection{Acknowledgements}

The initial impetus for this project came from Matt Dean; along the way substantial encouragement and useful suggestions were provided by Simon Tavaré, Rasmus Nielsen, Graham Coop, Yaniv Brandvain, and Sergey Nuzhdin. Many thanks are also due to Jessica Crisci for curating the human-chimp data. The majority of this work was done while PR was at USC.

FAM's work supported by NIH grants R01 AI146028 and U01 AI150747. Dr. Matsen is an Investigator of the Howard Hughes Medical Institute. PLR's work was supported by NIH award R01HG010774

\section{References}

L B Alexandrov, S Nik-Zainal, D C Wedge, P J Campbell, and M R Stratton. Deciphering signatures of mutational processes operative in human cancer. Cell Rep, 3(1):246-259, January 2013a. doi: 10.1016/j. celrep.2012.12.008. URL https://www.ncbi.nlm.nih.gov/pmc/articles/PMC3588146/.

Ludmil B. Alexandrov, Serena Nik-Zainal, David C. Wedge, Samuel A. J. R. Aparicio, Sam Behjati, Andrew V. Biankin, Graham R. Bignell, Niccolo Bolli, Ake Borg, Anne-Lise Borresen-Dale, Sandrine Boyault, Birgit Burkhardt, Adam P. Butler, Carlos Caldas, Helen R. Davies, Christine Desmedt, Roland Eils, Jorunn Erla Eyfjord, John A. Foekens, Mel Greaves, Fumie Hosoda, Barbara Hutter, Tomislav Ilicic, Sandrine Imbeaud, Marcin Imielinsk, Natalie Jager, David T. W. Jones, David Jones, Stian Knappskog, Marcel Kool, Sunil R. Lakhani, Carlos Lopez-Otin, Sancha Martin, Nikhil C. Munshi, Hiromi Nakamura, Paul A. Northcott, Marina Pajic, Elli Papaemmanuil, Angelo Paradiso, John V. Pearson, Xose S. Puente, Keiran Raine, Manasa Ramakrishna, Andrea L. Richardson, Julia Richter, Philip Rosenstiel, Matthias Schlesner, Ton N. Schumacher, Paul N. Span, Jon W. Teague, Yasushi Totoki, Andrew N. J. Tutt, Rafael Valdes-Mas, Marit M. van Buuren, Laura van /'t Veer, Anne Vincent-Salomon, Nicola Waddell, Lucy R. Yates, Australian Pancreatic Cancer Genome Initiative, ICGC Breast Cancer Consortium, ICGC MMML-Seq Consortium, ICGC PedBrain, Jessica Zucman-Rossi, P. Andrew Futreal, Ultan McDermott, Peter Lichter, Matthew Meyerson, Sean M. Grimmond, Reiner Siebert, Elias Campo, Tatsuhiro Shibata, Stefan M. Pfister, Peter J. Campbell, and Michael R. Stratton. Signatures of mutational processes in human cancer. Nature, 500(7463):415-421, August 2013b. ISSN 00280836. URL http://dx.doi.org/10.1038/nature12477.

Barbara Arbeithuber, Andrea J. Betancourt, Thomas Ebner, and Irene Tiemann-Boege. Crossovers are associated with mutation and biased gene conversion at recombination hotspots. Proceedings of the National Academy of Sciences, 112(7):2109-2114, 2015. doi: 10.1073/pnas.1416622112. URL http: //www.pnas.org/content/112/7/2109. abstract.

P F Arndt, C B Burge, and T Hwa. DNA sequence evolution with neighbor-dependent mutation. J Comput Biol, 10(3-4):313-322, 2003. doi: 10.1089/10665270360688039. URL http://www.ncbi.nlm.nih.gov/ pubmed/12935330. 
G Baele, Y Van de Peer, and S Vansteelandt. Using non-reversible context-dependent evolutionary models to study substitution patterns in primate non-coding sequences. J Mol Evol, 71(1):34-50, July 2010a. doi: 10.1007/s00239-010-9362-y. URL http://www.ncbi.nlm.nih.gov/pubmed/20623275.

Guy Baele, Yves Van de Peer, and Stijn Vansteelandt. Modelling the ancestral sequence distribution and model frequencies in context-dependent models for primate non-coding sequences. BMC Evolutionary Biology, 10(1):244, 2010b. ISSN 1471-2148. doi: 10.1186/1471-2148-10-244. URL http: //www.biomedcentral.com/1471-2148/10/244.

Jean Bérard and Laurent Guéguen. Accurate estimation of substitution rates with neighbor-dependent models in a phylogenetic context. Syst Biol, 61(3):510-521, May 2012. doi: 10.1093/sysbio/sys024. URL http://www.ncbi.nlm.nih.gov/pubmed/22331438?dopt=Abstract.

Julian E. Besag. Nearest-neighbour systems and the auto-logistic model for binary data. Journal of the Royal Statistical Society. Series B (Methodological), 34(1):pp. 75-83, 1972. ISSN 00359246. URL http: //www.jstor.org/stable/2985051.

Julian E. Besag. Spatial interaction and the statistical analysis of lattice systems. Journal of the Royal Statistical Society. Series B (Methodological), 36(2):pp. 192-236, 1974. ISSN 00359246. URL http: //www.jstor.org/stable/2984812.

Julian E. Besag. On the statistical analysis of dirty pictures. Journal of the Royal Statistical Society, 48(3): 259-302, 1986.

Anindya Bhadra, Jyotishka Datta, Nicholas G. Polson, and Brandon Willard. The horseshoe+ estimator of ultra-sparse signals, 2015. URL http://arxiv.org/abs/1502.00560. cite arxiv:1502.00560.

C Burge, A M Campbell, and S Karlin. Over- and under-representation of short oligonucleotides in DNA sequences. Proceedings of the National Academy of Sciences, 89(4):1358-1362, 1992. URL http://www. pnas. org/content/89/4/1358. abstract.

O F Christensen, A Hobolth, and J L Jensen. Pseudo-likelihood analysis of codon substitution models with neighbor-dependent rates. J Comput Biol, 12(9):1166-1182, November 2005. doi: 10.1089/cmb.2005.12. 1166. URL http://www.ncbi.nlm.nih.gov/pubmed/16305327.

Keith C Clarke, James A Brass, and Philip J Riggan. A cellular automation model of wildfire propagation and extinction. Photogrammetric Engineering and Remote Sensing, 60(11):1355-1367, 1994.

Sarah Cobey, Patrick Wilson, and Frederick A. Matsen. The evolution within us. Philosophical Transactions of the Royal Society of London B: Biological Sciences, 370(1676), 2015. ISSN 0962-8436. doi: 10.1098/ rstb.2014.0235. URL http://rstb.royalsocietypublishing.org/content/370/1676/20140235.

Francis Comets. On consistency of a class of estimators for exponential families of Markov random fields on the lattice. The Annals of Statistics, 20(1):455-468, 03 1992. doi: 10.1214/aos/1176348532. URL http://dx.doi.org/10.1214/aos/1176348532.

Laurent Duret and Nicolas Galtier. Biased gene conversion and the evolution of mammalian genomic landscapes. Annual Review of Genomics and Human Genetics, 10(1):285-311, 2009. doi: 10.1146/annurev-genom-082908-150001. URL http://www.annualreviews.org/doi/abs/10.1146/ annurev-genom-082908-150001. PMID: 19630562.

Joseph Felsenstein. Evolutionary trees from dna sequences: a maximum likelihood approach. Journal of molecular evolution, 17(6):368-376, 1981.

Joseph Felsenstein and Gary A Churchill. A hidden markov model approach to variation among sites in rate of evolution. Molecular biology and evolution, 13(1):93-104, 1996. 
Nial Friel. Bayesian inference for Gibbs random fields using composite likelihoods. In Proceedings of the Winter Simulation Conference, WSC '12, pages 28:1-28:8. Winter Simulation Conference, 2012. URL http://dl.acm.org/citation. cfm?id=2429759.2429795.

Arnoldo Frigessi and Mauro Piccioni. Parameter estimation for two-dimensional Ising fields corrupted by noise. Stochastic Processes and their Applications, 34(2):297 - 311, 1990. ISSN 0304-4149. doi: http: //dx.doi.org/10.1016/0304-4149(90)90020-S. URL http://www. sciencedirect.com/science/article/ pii/030441499090020S.

Ziyue Gao, Priya Moorjani, Thomas A. Sasani, Brent S. Pedersen, Aaron R. Quinlan, Lynn B. Jorde, Guy Amster, and Molly Przeworski. Overlooked roles of DNA damage and maternal age in generating human germline mutations. Proceedings of the National Academy of Sciences, 116(19):9491-9500, 2019. ISSN 0027-8424. doi: 10.1073/pnas.1901259116. URL https://www.pnas.org/content/116/19/9491.

A.E. Gelfand, M. Fuentes, P. Guttorp, and P. Diggle. Handbook of Spatial Statistics. Chapman \& Hall/CRC Handbooks of Modern Statistical Methods. Taylor \& Francis, 2010. ISBN 9781420072877. URL http: //books.google.com/books?id=EFbbcMFZ2mMC.

Stuart Geman and Donald Geman. Stochastic relaxation, Gibbs distributions, and the Bayesian restoration of images. Pattern Analysis and Machine Intelligence, IEEE Transactions on, PAMI-6(6):721-741, November 1984. ISSN 0162-8828. doi: 10.1109/TPAMI.1984.4767596. URL http://dl.acm.org/citation.cfm? id $=2286617$.

Charles J. Geyer and Leif T. Johnson. mcmc: Markov Chain Monte Carlo, 2017. URL https://CRAN. $\mathrm{R}$-project.org/package=mcmc. R package version 0.9-5.

Roy J. Glauber. Time-dependent statistics of the ising model. Journal of Mathematical Physics, 4(2), 1963. URL http://dx.doi.org/10.1063/1.1703954.

S Glémin, P F Arndt, P W Messer, D Petrov, N Galtier, and L Duret. Quantification of GC-biased gene conversion in the human genome. Genome Res, 25(8):1215-1228, August 2015. doi: 10.1101/gr.185488.114. URL https://www.ncbi.nlm.nih.gov/pubmed/25995268.

M F Goodman and R Woodgate. Translesion DNA polymerases. Cold Spring Harb Perspect Biol, 5(10), October 2013. doi: 10.1101/cshperspect.a010363. URL https://www.ncbi.nlm.nih.gov/pmc/articles/ PMC3783050/.

Myron F. Goodman. Error-prone repair DNA polymerases in prokaryotes and eukaryotes. Annual Review of Biochemistry, 71(1):17-50, 2002. doi: 10.1146/annurev.biochem.71.083101.124707. URL https://doi. org/10.1146/annurev. biochem.71.083101.124707.

Vincent Goulet, Christophe Dutang, Martin Maechler, David Firth, Marina Shapira, and Michael Stadelmann. expm: Matrix Exponential, Log, 'etc', 2017. URL https://CRAN.R-project.org/package=expm. $\mathrm{R}$ package version $0.999-2$.

Kelley Harris. Evidence for recent, population-specific evolution of the human mutation rate. Proceedings of the National Academy of Sciences, 112(11):3439-3444, 2015. doi: 10.1073/pnas.1418652112. URL http://www. pnas.org/content/112/11/3439. abstract.

Kelley Harris and Rasmus Nielsen. Error-prone polymerase activity causes multinucleotide mutations in humans, 2013. URL http://arxiv.org/abs/1312.1395. cite arxiv:1312.1395.

Kelley Harris and Jonathan Pritchard. Rapid evolution of the human mutation spectrum. bioRxiv, 2016. doi: 10.1101/084343. URL http://biorxiv.org/content/early/2016/10/30/084343.

Masami Hasegawa, Hirohisa Kishino, and Taka-aki Yano. Dating of the human-ape splitting by a molecular clock of mitochondrial dna. Journal of molecular evolution, 22(2):160-174, 1985. 
José María Heredia-Genestar, Tomàs Marquès-Bonet, David Juan, and Arcadi Navarro. Extreme differences between human germline and tumor mutation densities are driven by ancestral human-specific deviations. Nature Communications, 11(1):2512-, 2020. ISSN 20411723. doi: 10.1038/s41467-020-16296-4. URL https://doi.org/10.1038/s41467-020-16296-4.

Asger Hobolth. A Markov chain Monte Carlo expectation maximization algorithm for statistical analysis of DNA sequence evolution with neighbor-dependent substitution rates. Journal of Computational and Graphical Statistics, 17(1):138-162, 2008. URL http://www.daimi.au.dk/ asger/JCGS08.pdf.

Asger Hobolth and Eric A. Stone. Simulation from endpoint-conditioned, continuous-time Markov chains on a finite state space, with applications to molecular evolution. The Annals of Applied Statistics, 3(3), September 2009. doi: 10.1214/09-aoas247. URL https://doi.org/10.1214\%2F09-aoas247.

Dick G Hwang and Phil Green. Bayesian markov chain monte carlo sequence analysis reveals varying neutral substitution patterns in mammalian evolution. Proc. Natl. Acad. Sci. U. S. A., 101(39):1399414001, September 2004. ISSN 0027-8424. doi: 10.1073/pnas.0404142101. URL http://dx.doi.org/10. 1073/pnas. 0404142101.

TH Jukes and CR Cantor. Evolution of protein molecules, pp. 21-120 in mammalian protein metabolism, edited by munro hm, 1969 .

S. Kasar, J. Kim, R. Improgo, G. Tiao, P. Polak, N. Haradhvala, M. S. Lawrence, A. Kiezun, S. M. Fernandes, S. Bahl, C. Sougnez, S. Gabriel, E. S. Lander, H. T. Kim, G. Getz, and J. R. Brown. Whole-genome sequencing reveals activation-induced cytidine deaminase signatures during indolent chronic lymphocytic leukaemia evolution. Nature Communications, 6:8866-, December 2015. URL http://dx.doi.org/10. $1038 /$ ncomms 9866 .

W. J. Kent, C. W. Sugnet, T. S. Furey, K. M. Roskin, T. H. Pringle, A. M. Zahler, and a. D. Haussler. The human genome browser at UCSC. Genome Research, 12(6):996-1006, May 2002. doi: 10.1101/gr.229102. URL https://doi.org/10.1101\%2Fgr.229102.

Motoo Kimura. On the probability of fixation of mutant genes in a population. Genetics, 47:713-719, June 1962. URL https://www.ncbi.nlm.nih.gov/pubmed/14456043.

Kevin E. Langergraber, Kay Prüfer, Carolyn Rowney, Christophe Boesch, Catherine Crockford, Katie Fawcett, Eiji Inoue, Miho Inoue-Muruyama, John C. Mitani, Martin N. Muller, Martha M. Robbins, Grit Schubert, Tara S. Stoinski, Bence Viola, David Watts, Roman M. Wittig, Richard W. Wrangham, Klaus Zuberbühler, Svante Pääbo, and Linda Vigilant. Generation times in wild chimpanzees and gorillas suggest earlier divergence times in great ape and human evolution. Proceedings of the National Academy of Sciences, 109(39):15716-15721, 2012. doi: 10.1073/pnas.1211740109. URL http://www. pnas.org/content/109/39/15716. abstract.

F Larribe and P Fearnhead. On composite likelihoods in statistical genetics. Statistica Sinica, 21(1):43, 2011. URL http://www3.stat.sinica.edu.tw/statistica/oldpdf/A21n12.pdf.

T.M. Liggett. Interacting particle systems. Springer Berlin Heidelberg, 2005.

Gerton Lunter and Jotun Hein. A nucleotide substitution model with nearest-neighbour interactions. Bioinformatics, 20 Suppl 1:216-223, August 2004. doi: 10.1093/bioinformatics/bth901. URL http: //www.ncbi.nlm.nih.gov/pubmed/15262802?dopt=Abstract.

Iain Mathieson and David Reich. Differences in the rare variant spectrum among human populations. PLOS Genetics, 13(2):1-17, 02 2017. doi: 10.1371/journal.pgen.1006581. URL https://doi.org/10.1371/ journal.pgen.1006581. 
R W Maul, T MacCarthy, E G Frank, K A Donigan, M P McLenigan, W Yang, H Saribasak, D E Huston, S S Lange, R Woodgate, and P J Gearhart. DNA polymerase $\iota$ functions in the generation of tandem mutations during somatic hypermutation of antibody genes. J Exp Med, 213(9):1675-1683, August 2016. doi: 10.1084/jem.20151227. URL https://www.ncbi.nlm.nih.gov/pubmed/27455952.

S Myers, R Bowden, A Tumian, R E Bontrop, C Freeman, T S MacFie, G McVean, and P Donnelly. Drive against hotspot motifs in primates implicates the PRDM9 gene in meiotic recombination. Science, 327 (5967):876-879, February 2010. doi: 10.1126/science.1182363. URL https://www.ncbi.nlm.nih.gov/ pubmed/20044541.

J. Neyman and E. S. Pearson. On the problem of the most efficient tests of statistical hypotheses. Philosophical Transactions of the Royal Society of London. Series A, Containing Papers of a Mathematical or Physical Character, 231:289-337, 1933. ISSN 02643952. URL http://www.jstor.org/stable/91247.

Anne-Mette Krabbe Pedersen and Jens Ledet Jensen. A dependent rates model and MCMC based methodology for the maximum likelihood analysis of sequences with overlapping reading frames, 2000. URL http://math.au.dk/en/research/publications/publication-series/publication/publid/187/.

G P Pfeifer. Mutagenesis at methylated CpG sequences. Curr Top Microbiol Immunol, 301:259-281, 2006. URL https://www.ncbi.nlm.nih.gov/pubmed/16570852.

David K. Pickard. Inference for general Ising models. Journal of Applied Probability, 19:pp. 345-357, 1982. ISSN 00219002. URL http://www.jstor.org/stable/3213574.

Steven A Roberts and Dmitry A Gordenin. Hypermutation in human cancer genomes: footprints and mechanisms. Nature reviews. Cancer, 14(12):786-800, December 2014. ISSN 14741768. URL http: //www.ncbi.nlm.nih.gov/pmc/articles/PMC4280484/.

Miguel Rodriguez-Galindo, Sònia Casillas, Donate Weghorn, and Antonio Barbadilla. Germline de novo mutation rates on exons versus introns in humans. Nature Communications, 11(1):3304-, 2020. ISSN 20411723. doi: 10.1038/s41467-020-17162-z. URL https://doi.org/10.1038/s41467-020-17162-z.

J E Sale, A R Lehmann, and R Woodgate. Y-family DNA polymerases and their role in tolerance of cellular DNA damage. Nat Rev Mol Cell Biol, 13(3):141-152, February 2012. doi: 10.1038/nrm3289. URL https://www.ncbi.nlm.nih.gov/pmc/articles/PMC3630503/.

C T Saunders and P Green. Insights from modeling protein evolution with context-dependent mutation and asymmetric amino acid selection. Mol Biol Evol, 24(12):2632-2647, December 2007. doi: 10.1093/molbev/ msm190. URL http://www.ncbi.nlm.nih.gov/pubmed/17906001.

A Scally, J Y Dutheil, L W Hillier, G E Jordan, I Goodhead, J Herrero, A Hobolth, T Lappalainen, T Mailund, T Marques-Bonet, S McCarthy, S H Montgomery, P C Schwalie, Y A Tang, M C Ward, Y Xue, B Yngvadottir, C Alkan, L N Andersen, Q Ayub, E V Ball, K Beal, B J Bradley, Y Chen, C M Clee, S Fitzgerald, T A Graves, Y Gu, P Heath, A Heger, E Karakoc, A Kolb-Kokocinski, G K Laird, G Lunter, S Meader, M Mort, J C Mullikin, K Munch, T D O'Connor, A D Phillips, J Prado-Martinez, A S Rogers, S Sajjadian, D Schmidt, K Shaw, J T Simpson, P D Stenson, D J Turner, L Vigilant, A J Vilella, W Whitener, B Zhu, D N Cooper, P de Jong, E T Dermitzakis, E E Eichler, P Flicek, N Goldman, N I Mundy, Z Ning, D T Odom, C P Ponting, M A Quail, O A Ryder, S M Searle, W C Warren, R K Wilson, M H Schierup, J Rogers, C Tyler-Smith, and R Durbin. Insights into hominid evolution from the gorilla genome sequence. Nature, 483(7388):169-175, March 2012. doi: 10.1038/nature10842. URL https://www.ncbi.nlm.nih.gov/pubmed/22398555.

V M Schaibley, M Zawistowski, D Wegmann, M G Ehm, M R Nelson, P L St Jean, G R Abecasis, J Novembre, S Zöllner, and J Z Li. The influence of genomic context on mutation patterns in the human genome inferred from rare variants. Genome Res, November 2013. doi: 10.1101/gr.154971.113. URL http: //www.ncbi.nlm.nih.gov/pubmed/23990608?dopt=Abstract. 
D R Schrider, J N Hourmozdi, and M W Hahn. Pervasive multinucleotide mutational events in eukaryotes. Curr Biol, 21(12):1051-1054, June 2011. doi: 10.1016/j.cub.2011.05.013. URL http://www.ncbi.nlm . nih.gov/pubmed/21636278.

Gunter M Schütz. Exact solution of the master equation for the asymmetric exclusion process. Journal of statistical physics, 88(1):427-445, 1997.

Gayan Senavirathne, Jeffrey G. Bertram, Malgorzata Jaszczur, Kathy R. Chaurasiya, Phuong Pham, Chi H. Mak, Myron F. Goodman, and David Rueda. Activation-induced deoxycytidine deaminase (aid) cotranscriptional scanning at single-molecule resolution. Nature Communications, 6:10209-, December 2015. URL http://dx.doi.org/10.1038/ncomms 10209.

Vladimir B. Seplyarskiy, Ruslan A. Soldatov, Evan Koch, Ryan J. McGinty, Jakob M. Goldmann, Ryan D. Hernandez, Kathleen Barnes, Adolfo Correa, Esteban G. Burchard, Patrick T. Ellinor, Stephen T. McGarvey, Braxton D. Mitchell, Ramachandran S. Vasan, Susan Redline, Edwin Silverman, Scott T. Weiss, Donna K. Arnett, John Blangero, Eric Boerwinkle, Jiang He, Courtney Montgomery, D.C. Rao, Jerome I. Rotter, Kent D. Taylor, Jennifer A Brody, Yii-Der Ida Chen, Lisa de las Fuentes, Chii-Min Hwu, Stephen S. Rich, Ani W. Manichaikul, Josyf C. Mychaleckyj, Nicholette D. Palmer, Jennifer A. Smith, Sharon L.R. Kardia, Patricia A. Peyser, Lawrence F. Bielak, Timothy D. O'Connor, Leslie S. Emery, NHLBI TransOmics for Precision Medicine (TOPMed) Consortium, TOPMed Population Genetics Working Group, Christian Gilissen, Wendy S. W. Wong, Peter V. Kharchenko, and Shamil Sunyaev. Population sequencing data reveal a compendium of mutational processes in the human germ line. Science, 2021. ISSN 0036-8075. doi: 10.1126/science.aba7408. URL https://science.sciencemag.org/content/early/ 2021/08/11/science. aba7408.

Yuichi Shiraishi, Georg Tremmel, Satoru Miyano, and Matthew Stephens. A simple model-based approach to inferring and visualizing cancer mutation signatures. PLOS Genetics, 11(12):1-21, 12 2015. doi: 10.1371/journal.pgen.1005657. URL https://doi.org/10.1371/journal.pgen.1005657.

A Siepel and D Haussler. Phylogenetic estimation of context-dependent substitution rates by maximum likelihood. Mol Biol Evol, 21(3):468-488, March 2004. doi: 10.1093/molbev/msh039. URL http://www . ncbi.nlm.nih.gov/pubmed/14660683.

Rajeshwar P. Sinha and Donat-P. Hader. UV-induced DNA damage and repair: a review. Photochem. Photobiol. Sci., 1(4):225-236, 2002. doi: 10.1039/B201230H. URL http://dx.doi.org/10.1039/B201230H.

G Teng and F N Papavasiliou. Immunoglobulin somatic hypermutation. Annu Rev Genet, 41:107-120, 2007. doi: 10.1146/annurev.genet.41.110306.130340. URL https://www.ncbi.nlm.nih.gov/pubmed/ 17576170 .

N V Terekhanova, G A Bazykin, A Neverov, A S Kondrashov, and V B Seplyarskiy. Prevalence of multinucleotide replacements in evolution of primates and Drosophila. Mol Biol Evol, 30(6):1315-1325, June 2013. doi: 10.1093/molbev/mst036. URL http://www.ncbi.nlm.nih.gov/pmc/articles/PMC3649671/.

Jeffrey L Thorne. Protein evolution constraints and model-based techniques to study them. Current Opinion in Structural Biology, 17(3):337 - 341, 2007. ISSN 0959-440X. doi: 10.1016/j.sbi.2007.05.006. URL http: //www.sciencedirect.com/science/article/pii/S0959440X07000711. Nucleic acids / Sequences and topology.

Fulong Wu. Calibration of stochastic cellular automata: the application to rural-urban land conversions. International Journal of Geographical Information Science, 16(8):795-818, 2002.

Gur Yaari, Jason Vander Heiden, Mohamed Uduman, Daniel Gadala-Maria, Namita Gupta, Joel N.H. Stern, Kevin O'Connor, David Hafler, Uri Laserson, Francois Vigneault, and Steven Kleinstein. Models of somatic hypermutation targeting and substitution based on synonymous mutations from high-throughput 
immunoglobulin sequencing data. Frontiers in Immunology, 4(358), 2013. ISSN 1664-3224. doi: 10.3389/ fimmu.2013.00358. URL http://www.frontiersin.org/b_cell_biology/10.3389/fimmu.2013.00358/ abstract.

Ziheng Yang. Maximum likelihood phylogenetic estimation from DNA sequences with variable rates over sites: approximate methods. J Mol Evol, 39(3):306-314, September 1994. URL https://www.ncbi.nlm. nih.gov/pubmed/7932792.

D R Zerbino, S P Wilder, N Johnson, T Juettemann, and P R Flicek. The ensembl regulatory build. Genome Biol, 16:56-56, 2015. doi: 10.1186/s13059-015-0621-5. URL http://www.ncbi.nlm.nih.gov/ pubmed/25887522.

Rita Maria Zorzenon dos Santos and Sérgio Coutinho. Dynamics of HIV infection: A cellular automata approach. Phys. Rev. Lett., 87(16):168102, September 2001. doi: 10.1103/PhysRevLett.87.168102. URL http://link.aps.org/doi/10.1103/PhysRevLett.87.168102. 


\section{A Supplementary figures}

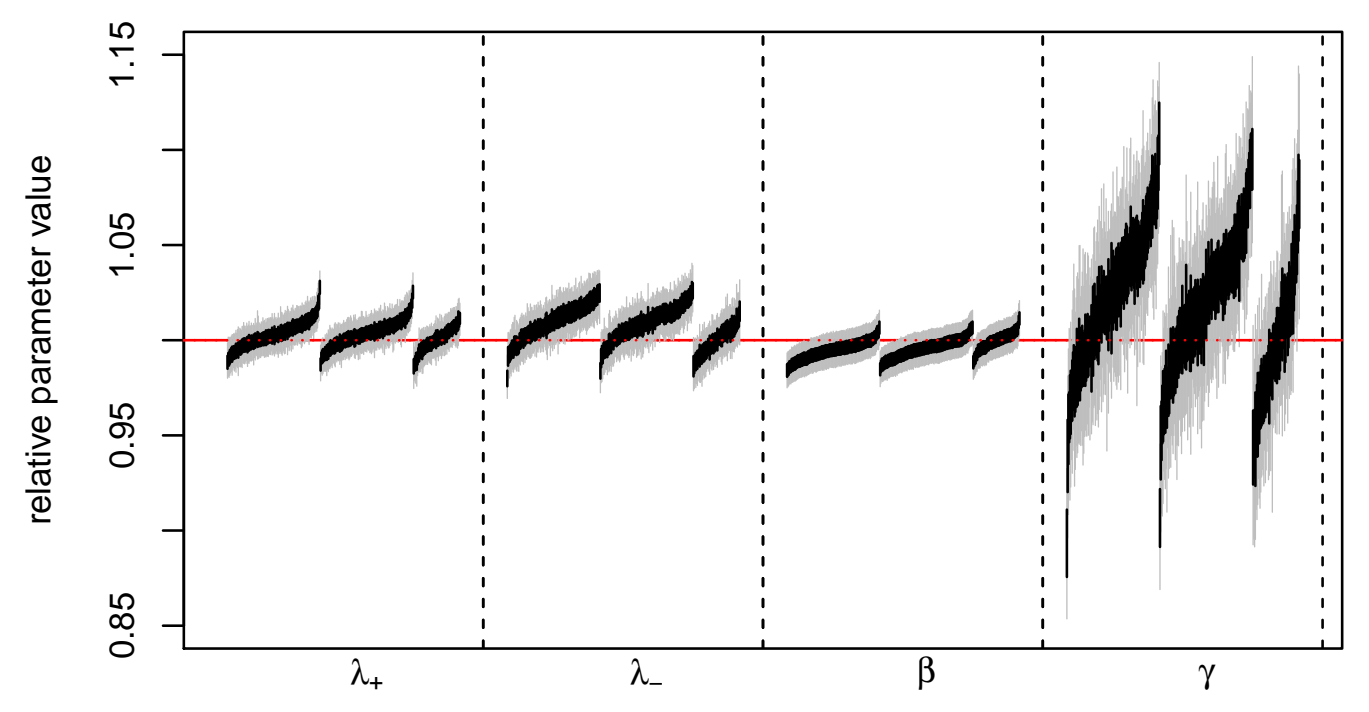

Figure S1: As in Figure 4: Credible intervals for the four parameters of the dynamic Ising model, from MCMC runs on independently simulated sequences of $10^{6}$ bases differing at around $18.6 \%$ of the sites. Shown are relative intervals, obtained by dividing by the true value. The three groups are left to right, credible intervals obtained from $(4,2,1),(5,3,1)$, and $(6,2,2)$ T-mers: note that only the longest $(6,2,2) \mathrm{T}$-mers provide well-calibrated coverage (Table 4 ) and do not show bias. 




- nongene : CTCF_binding_site - nongene : enhancer - nongene : open_chromatin_region - nongene : promoter_flanking_region - gene : CTCF_binding_site - gene : enhancer

- gene : open chromatin region - gene : promoter_flanking_region

Figure S2: As in Figure 5, but with reduced vertical axis range to see in more detail: estimated average mutation rates for the motifs in the "Hominid data" section since the common ancestor of humans and chimpanzee. Rates are scaled so that the mean time to common ancestor is 0.001 units - if we take this to be 6 million years [Scally et al., 2012] then rates are in units of mutations per 6,000 years. Shown are posterior medians (points) and 95\% credible intervals (lines). 
bioRxiv preprint doi: https://doi.org/10.1101/2021.12.15.472813; this version posted December 16, 2021. The copyright holder for this preprint (which was not certified by peer review) is the author/funder, who has granted bioRxiv a license to display the preprint in perpetuity. It is made available under aCC-BY 4.0 International license.

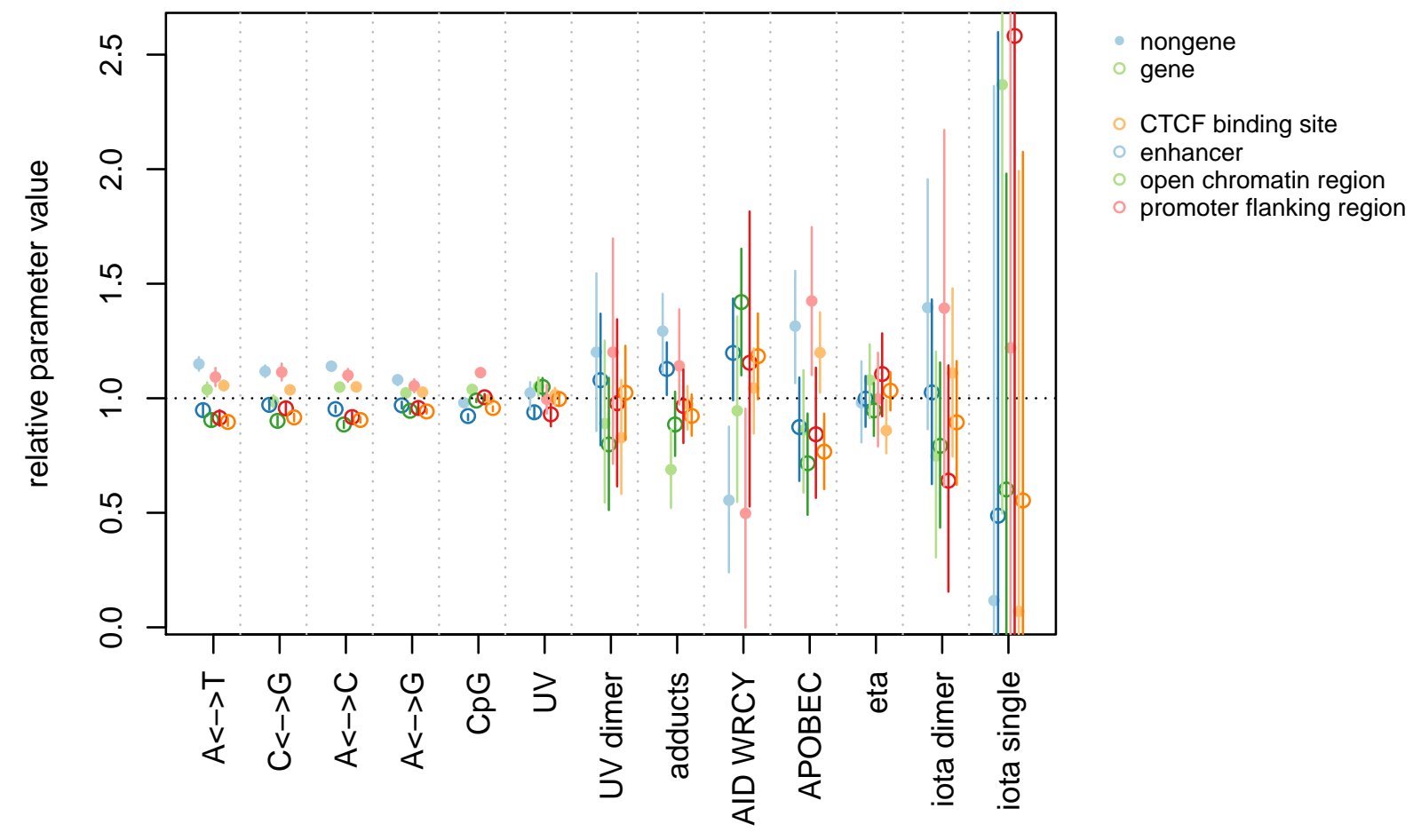

Figure S3: As in Figures 5 and S2, but where each rate is divided by the mean rate across all eight categories (as well as the $95 \%$ credible interval endpoints). 


\section{B Proof of the approximation}

First we quantify and prove the approximation (4). The basic idea is that the probability is only in error if there is a chain of mutations that create a dependency between the base of the T-mer and the flanking sequence. To simplify the argument, first assume that all transition triples change only a single, central site in the pattern, taking into account context on each side of maximum length $w$. (For instance, a trinucleotide model in which the flanking sites on each side affect the mutation rate of the central site has $w=1$.)

Recall that, as described in the "Simulation" section, we may represent the process by first laying down a homogeneous Poisson process with rate $\mu^{*}$ per site of "potential mutations", where $\mu^{*}$ is the maximum possible mutation rate, and then resolving these appropriately. (In particular, the times and locations of these potential mutations are chosen uniformly.) Suppose a possible change occurs at site $i-w$, at time $t$, which therefore might result in a change in mutation rate at site $i$. To resolve this, we need to know the outcome of any other potential changes that might change the sequence between $i-w$ and $i+w$. Consider those that might happen only to the right of $i$, thus extending the sequence on which the change depends to the right. The number of such potential changes is Poisson with mean $\mu^{*} w t$; if this number is nonzero, then this extends the amount of sequence on which the change at site $i$ depends by a uniform number between 1 and $w$. If one occurs, say at time $0<s<t$, then any further potential changes that occur between time 0 and time $s$ in the $w$ sites to the right of this older potential change will again extend the range of dependency. Putting this together, the amount of sequence to the right of a site on which a mutation at that site depends is bounded by the compound Poisson process $\sum_{k=1}^{N} W_{k}$, where $N$ is Poisson with mean $\mu^{*} w t$ and each $W_{k}$ is independent and uniform on $\{1,2, \ldots, w\}$.

Now consider the probability of interest,

$$
\mathbb{P}\left\{X_{i+\ell}^{(n-\ell-r)}(t)=y \mid X_{i}^{(n)}(0)=x\right\} .
$$

This probability can be written as a sum over all possible arrangements and resolutions of potential changes. Our approximation, $p_{n, \ell, r}(x, y)$ is the probability that would be obtained by ignoring any potential changes that rely on context outside of the $n$-sequence $x$. So, if $A$ is the event that the final subsequence $y$ depends on sequence outside of $x$ due to potential changes, then

$$
\left|\mathbb{P}\left\{X_{i+\ell}^{(n-\ell-r)}(t)=y \mid X_{i}^{(n)}(0)=x\right\}-p_{n, \ell, r}(x, y)\right| \leq \mathbb{P}\{A\} .
$$

Since the size of the dependency window is a compound Poisson process, we can bound $\mathbb{P}\{A\}$ using the following lemma:

Lemma 1. Let $V=\sum_{j=1}^{N} W_{j}$, where $N$ is Poisson with mean $\lambda$ and each $W_{j}$ is independent and bounded between 0 and $w$. Then for $k \geq 1$,

$$
\mathbb{P}\{V>k w\} \leq \frac{\lambda^{k+1}}{(k+1) !} .
$$

Proof. First note that $\mathbb{P}\{V>k w\} \leq \mathbb{P}\{N>k\}$. Now, use the fact that if $f(n)=n(n-1) \cdots(n-k) /(k+1)$ ! then $f(n)=0$ for integers $0 \leq n \leq k$ and $f(n) \geq 1$ for $n>k$, and so $\mathbb{P}\{N>k\} \leq \mathbb{E}[f(N)]$. Now, $\mathbb{E}[f(N)]=\lambda^{k+1} /(k+1)$ ! via direct calculation or the formula for the $k+1$ st factorial moment for the Poisson distribution.

Putting this together, we find that

$$
\left|\mathbb{P}\left\{X_{i+\ell}^{(n-\ell-r)}(t)=y \mid X_{i}^{(n)}(0)=x\right\}-p_{n, \ell, r}(x, y)\right| \leq \frac{2\left(\mu^{*} t\right)^{k+1}}{(k+1) !} .
$$

Here, the factor of 2 comes from the two sides (to the left and right) of the base of the T-mer.

The argument applies to more general transition triples, with $w$ set equal to the maximum distance from a position that is changed to the edge of the context across all transition triples. 


\section{Pruning algorithm}

Fix one tip of the tree, $a$, to be the taxa where we count "long" sequences, let $\rho$ be the root, and let $b_{1}, \ldots, b_{n}$ be the remaining tips, where we observe "short" sequences. Let $v_{0}=\rho, v_{1}, \ldots, v_{\ell}=a$ be the path from the root down to $a$, let $u_{1}, u_{2}, \ldots, u_{m}$ be the remaining internal nodes, and for each $v_{i}$ let $u\left(v_{i}\right)$ be the offspring of $v_{i}$ that is not one of the other $v$. For each node in the tree, we will compute a matrix of probabilities: for nodes not on the path from $a$ to the root, we compute the chance that that each "long" sequence observed at that node is matched by each possible combination of "short" sequences on the tips of the tree below it. For nodes on the path from $a$ to the root, we compute the probability of observing each "long" sequence at that node, along with the combinations of "short" sequences on all tips further away from $a$ (i.e., below that node if it was rooted at $a$ ). For a node $w$ this will be stored as a $|\mathcal{S}|^{n} \times|\mathcal{S}|^{c m}$ matrix, denoted $M(w)$, where $c$ is the number of tips for which $w$ lies on the path from $a$ to the tip. Rows of $M\left(u_{j}\right)$ sum to 1 , while each entire matrix $M\left(v_{i}\right)$ sums to 1 ; the difference being that $M\left(v_{i}\right)$ sums over the prior at the root, and $M\left(u_{j}\right)$ takes the value at $u_{j}$ as given. Below, we index columns of $M(u)$ by lists of short patterns, and leave the specific ordering to implementation. (For instance, if $c=2$ then a generic entry is $M(u)_{x,(y, z)}$, where the long pattern $x$ indexes the rows and the pair of short patterns $(y, z)$ indexes the columns; in the software all matrices retain column names making this unambiguous.)

Let $t(w)$ be the length of the branch above node $w$, for $w \neq \rho$, and denote by $G^{T}$ the transpose of $G$.

1. Set $M\left(b_{i}\right)=U$ for each $b_{i}$.

2. While there are $u_{j}$ whose children $w_{1}$ and $w_{2}$ both have matrices computed, let

$$
M\left(u_{j}\right)_{x,(y, z)}=\left(e^{t\left(w_{1}\right) G} M\left(w_{1}\right)\right)_{x, y}\left(e^{t\left(w_{2}\right) G} M\left(w_{2}\right)\right)_{x, z} .
$$

3. Let

$$
M(\rho)_{x, y}=\pi(x)\left(e^{t(u(\rho)) G} M(w(\rho))\right)_{x, y} .
$$

4. For each $1 \leq k<\ell$, let

$$
M\left(v_{k}\right)_{x,(y, z)}=\left(e^{t\left(v_{k}\right) G^{T}} M\left(v_{k-1}\right)\right)_{y, x}\left(e^{t\left(u\left(v_{k}\right)\right) G} M\left(u\left(v_{k-1}\right)\right)\right)_{x, z} .
$$

5. Finally, let

$$
M(a)_{x, y}=\left(e^{t(a) G^{T}} M\left(v_{\ell-1}\right)\right)_{x, y}
$$

